\title{
Article \\ Inhibition of Several Bacterial Species Isolated from Squid and Shrimp Skewers by Different Natural Edible Compounds
}

\author{
Lourenço Pinto de Rezende $^{1} \mathbb{D}$, Joana Bastos Barbosa ${ }^{1, * \mathbb{D}}$, Ana Maria Gomes ${ }^{1} \mathbb{D}$, Ana Machado Silva ${ }^{2}$, \\ Daniela Fonseca Correia ${ }^{2}$ and Paula Teixeira ${ }^{1}$ (D)
}

1 Laboratório Associado, Escola Superior de Biotecnologia, CBQF-Centro de Biotecnologia e Química Fina, Universidade Católica Portuguesa, 4169-005 Porto, Portugal; pinto.rezende88@gmail.com (L.P.d.R.); amgomes@ucp.pt (A.M.G.); pcteixeira@ucp.pt (P.T.)

2 SONAE, Lugar do Espido, Via Norte, 4471-909 Maia, Portugal; amsilva@sonaemc.com (A.M.S.); dafcorreia@sonaemc.com (D.F.C.)

* Correspondence: jbarbosa@ucp.pt

check for

updates

Citation: Pinto de Rezende, L.; Barbosa, J.B.; Gomes, A.M.; Silva, A.M.; Correia, D.F.; Teixeira, P. Inhibition of Several Bacterial Species Isolated from Squid and Shrimp Skewers by Different Natural Edible Compounds. Foods 2022, 11, 757. https://doi.org/10.3390/foods 11050757

Academic Editors: Patrícia Anacleto and Soottawat Benjakul

Received: 31 December 2021

Accepted: 3 March 2022

Published: 6 March 2022

Publisher's Note: MDPI stays neutral with regard to jurisdictional claims in published maps and institutional affiliations.

Copyright: (C) 2022 by the authors. Licensee MDPI, Basel, Switzerland. This article is an open access article distributed under the terms and conditions of the Creative Commons Attribution (CC BY) license (https:// creativecommons.org/licenses/by/ $4.0 /)$.

\begin{abstract}
Seafood is an excellent source of nutrients, essential for a healthy diet, ranging from proteins and fatty acids to vitamins and minerals. Seafood products are highly perishable foods due to their nutritional characteristics and composition. The application of nontoxic, natural, and edible preservatives to extend the shelf-life and inhibit bacterial proliferation of several foods has been a hot topic. Consequently, this work aimed to perform the microbiological characterization of squid and shrimp skewers during their shelf-life (five days) and evaluate the susceptibility of randomly isolated microorganisms to several natural edible compounds so that their application for the preservation and shelf-life extension of the product might be analyzed in the future. The product had considerably high total microorganisms loads of about $5 \log \mathrm{CFU} / \mathrm{g}$ at day zero and $9 \mathrm{log}$ CFU/g at day five. In addition, high bacterial counts of Pseudomonas spp., Enterobacterales, and lactic acid bacteria (LAB) were found, especially on the last day of storage, being Pseudomonas the dominant genus. However, no Escherichia coli or Listeria monocytogenes were detected on the analyzed samples. One hundred bacterial isolates were randomly selected and identified through 16s rRNA sequencing, resulting in the detection of several Enterobacterales, Pseudomonas spp., and LAB. The antibacterial activity of carvacrol, olive leaf extract, limonene, Citrox ${ }^{\circledR}$, different chitosans, and ethanolic propolis extracts was evaluated by the agar diffusion method, and the minimum inhibitory concentration was determined only for Citrox ${ }^{\circledR}$ since only this solution could inhibit all the identified isolates. At concentrations higher than or equal to $1.69 \%(v / v)$, Citrox ${ }^{\circledR}$ demonstrated bacteriostatic and bactericidal activity to $97 \%$ and $3 \%$ of the isolates, respectively. To our knowledge, there are no available data about the effectiveness of this commercial product on seafood isolates. Although preliminary, this study showed evidence that Citrox ${ }^{\circledR}$ has the potential to be used as a natural preservative in these seafood products, improving food safety and quality while reducing waste. However, further studies are required, such as developing a Citrox ${ }^{\circledR}$-based coating and its application on this matrix to validate its antimicrobial effect.
\end{abstract}

Keywords: antimicrobial activity; $\mathrm{Citrox}^{\circledR}$; seafood products; spoilage

\section{Introduction}

Seafood is regarded as a great source of nutrients, essential for a healthy diet, ranging from proteins and fatty acids to vitamins and minerals [1]. Due to this, there is an increasing demand for new, fresh, precooked, and ready-to-eat seafood products [2]. However, their nutritional characteristics and composition turn seafood products into highly perishable foods. The resulting degradation leads to large quantities of product waste [3], culminating in financial losses and quality concerns for both the industry and the consumers [1].

The degradation of fish products by the activity of specific spoilage organisms (SSOs) is the most concerning cause of spoilage of products faced by the producers and retailers [4]. 
Particular species of spoilage organisms can vary depending on the origin of the product and the processing techniques employed during its handling and storage [5]. In the case of seafood products, most of the spoiling agents are Gram-negative psychrotolerant bacteria. Of these, there is an over-representation of genera belonging to the phylum of Proteobacteria [6], such as Pseudomonas, Vibrio, Moraxella, Shewanella, Acinetobacter, Aeromonas, and Photobacterium. Gram-positive bacteria of the genera Micrococcus and Clostridium, as well as lactic acid bacteria (LAB), can also be present [6,7]. In shellfish, high concentrations of nonprotein nitrogen compounds and $\mathrm{pH}$ values typically above 6.0 represent excellent conditions for the proliferation of SSOs of the Morganella, Proteus, and Pseudomonas genera [7].

The production of chemical compounds such as trimethylamine, ammonia, hydrogen sulphide, methylmercaptan, and dimethyl-disulphide from the reduction of trimethylamine oxide, the metabolization of urea or deamination of amino acids, and the breakdown of sulphurous compounds are typical consequences of the proliferation of bacteria such as Shewanella spp., LAB, and Photobacterium spp. [6]. Additionally, the production of biogenic amines, such as cadaverine, putrescine, and histamine, can be induced by the decarboxylation of amino acids by bacteria such as Shewanella putrefaciens, Hafnia alvei, and Morganella morganii [6].

The growth of pathogenic microorganisms in food products also demands great attention. Seafood-associated illnesses have been linked to a variety of viruses, bacteria, and parasites [8]. The presence of Vibrio spp., Clostridium spp., Salmonella spp., Shigella spp., and some strains of Staphylococcus aureus can induce severe disease and is of paramount importance when evaluating whether a product is safe for consumption or not. These pathogens are primarily present in food products due to the contamination and pollution of the environments from which the product originated [8]. Moreover, contamination of food products with pathogens, resulting from the cross-contamination of products and/or from the migration of microorganisms from the gastrointestinal tracts, such as Listeria monocytogenes [9] and some strains of Escherichia coli, is typically the result of poor handling practices in food processing $[10,11]$. This intestine to muscle migration can occur if the period between death and evisceration of the animal is too long [9]. In addition to resulting in loss of nutritional value, the presence of pathogens in food products can also lead to serious foodborne illnesses [12].

Due to increasing consumer demands to reduce the use of synthetic chemicals in food, intense pressure to innovate and to provide natural alternatives has been felt all over the food industry [13]. The application of nontoxic, edible preservatives to extend shelf-life and inhibit bacterial proliferation in seafood has been the focus of several recent studies [14-16]. These preservatives have the potential to retard the spoilage of the product, retaining the hedonic characteristics of seafood, such as smell, texture, and flavor [16]. Natural antimicrobials, such as limonene, thymol, oleuropein, carvacrol, propolis, and chitosan, present valuable alternatives to the undesirable synthetic antimicrobials [15-20]. The nonsynthetic nature of these compounds provides the treated products with an allnatural characteristic, contributing to a more significant appeasement of consumer interests. For a product to be accepted as a potential additive to food products, every component should be food-grade. Thus, no safety issue should arise when using the product under the recommended conditions. On account of the inherent safety of food-grade products, natural and edible solutions can be applied to most food products to extend their shelf-life [21]. As a consequence of their popularity as promising innovations, numerous components demonstrating antimicrobial activity have been incorporated into these edible solutions with positive results $[15,16,22]$.

This work focused on analyzing the antibacterial activity of several natural compounds against the proliferation of bacteria isolated from uncooked seafood products consisting of squid (Loligo duvauceli) and shrimp (Parapenaeopsis, Penaeus, and Metapenaeus genera). Being this an extremely perishable food product, the inhibition of bacteria collected from the product with nonsynthetic, natural, and edible compounds can open the way to the 
formulation of efficient and safe coatings and treatments, capable of extending the shelf-life of such perishable products while maintaining an all-natural label.

\section{Materials and Methods}

\subsection{Microbiological Characterisation of Squids and Shrimps Skewers}

The product (two units of each of two lots) was transported to the laboratory under refrigeration conditions and analyzed immediately and after 2 and 5 days of refrigerated storage (stored at $4{ }^{\circ} \mathrm{C}$ and at not controlled atmospheric conditions in the original plastic boxes). Due to a shelf-life of 3 to 4 days, such periods were selected to represent a fresh product, one halfway through its shelf-life limit, and another after its shelf-life. This shelf-life period is a result of the sensorial perception of degradation observed during storage, being the product's organoleptic characteristics unfit for consumption after 4 days of refrigerated storage.

To achieve reproducibility and representative samples, two units of each lot were mixed to perform a composite sample. Each sample was composed of $10 \mathrm{~g}$ of squid mantle, $5 \mathrm{~g}$ of squid tentacles, and $10 \mathrm{~g}$ of shrimp meat (with no exoskeleton). According to standard microbiological methods, each of these $25 \mathrm{~g}$ samples were added to $225 \mathrm{~mL}$ of sterile buffered peptone water (Biokar Diagnostics, Beauvais, France) and homogenized in a stomacher (Interscience, Saint Nom la Brèteche, France) for 2 min. Appropriate decimal dilutions were prepared in Ringer's solution (Biokar Diagnostics) for microbial enumeration: colony counts of viable microorganisms at $30^{\circ} \mathrm{C}$ for $72 \mathrm{~h}$ on Plate Count Agar (PCA, Biokar Diagnostics; ISO 4833-1 [23]); LAB at $30^{\circ} \mathrm{C}$ for $72 \mathrm{~h}$ on de Man-Rogosa and Sharpe agar (MRSA, Biokar Diagnosis; ISO 15214 [24]); Pseudomonas spp. counts at $30^{\circ} \mathrm{C}$ for $48 \mathrm{~h}$ on Pseudomonas Agar Base (PAB, Biokar Diagnostics, ISO 13720 [25]) supplemented with cetrimide, fusidic acid and cefaloridin (VWR, Alfragide, Portugal); Enterobacterales at $37^{\circ} \mathrm{C}$ for $24 \mathrm{~h}$ on RAPID' Enterobacteriaceae medium (Bio-Rad, Hercules, CA, USA; ISO 21528 [26]); Listeria monocytogenes at $37^{\circ} \mathrm{C}$ for $24 \mathrm{~h}$ on Agar Listeria Ottaviani and Agosti (ALOA, Biokar Diagnosis, ISO 11290-2 [27]); Escherichia coli at $44^{\circ} \mathrm{C}$ for $24 \mathrm{~h}$ on Tryptone Bile X-Glucuronide Medium (TBX, bioMérieux; ISO 16649-1 [28]). Since the recommended storage of the product is at refrigeration temperatures, bacterial enumeration was also performed in all previously mentioned growth culture media at $11^{\circ} \mathrm{C}$.

\subsection{Selection of Isolates}

After enumeration, about $10 \%$ of colonies from each countable dilution plate were randomly selected. Presumptively, only presumptive colonies of Pseudomonas spp. and LAB were confirmed since the other microorganisms were grown on chromogenic (RAPID' Enterobacteriaceae and TBX) or nonselective (PCA) culture media. Characteristic colonies in terms of color, type, elevation, and opacity were confirmed by Gram staining and oxidase test (Pseudomonas spp.) and Gram staining and catalase test (LAB).

Each randomly selected colony was purified by repeated streaking onto the general growth medium TSAYE-Trypticase Soy Agar (Biokar Diagnostics) with $0.6 \%$ Yeast Extract (Biokar Diagnostics) or MRSA (in the case of LAB) and incubated for 24 to $72 \mathrm{~h}$ under the same conditions. All isolates $(10 \%)$ recovered were stored at $-80^{\circ} \mathrm{C}$ in Tryptic Soy Broth (TSB, Pronadisa) or MRS broth (only LAB) with 30\% (v/v) of glycerol (Sigma, Steinheim, Germany) and subcultured twice before use.

\subsection{Identification of Selected Isolates by $16 S$ rRNA Sequencing}

Each selected isolate was identified by $16 \mathrm{~S}$ rRNA sequencing. The DNA was extracted according to the protocols for total DNA purification from Gram-positive or -negative bacteria of the GRS genomic DNA Kit (Grisp, Porto, Portugal). The primers used for the amplification of the gene fragments for the 16s rRNA identification were 27F ( $5^{\prime}-$ AGAGTTTGATCCTGGCTCAG-3') and 1492R (5'-GGTTACCTTGTTACGACTT-3') [29]. For the preparation of the master mix, the following solutions were used: dNTP $(10 \mathrm{mM})$, Taq buffer $\mathrm{KCl}(10 \times), \mathrm{MgCl} 2(25 \mathrm{mM})$, primers $27 \mathrm{~F}$ and 1492R (100 $\mu \mathrm{M}$ each), Taq polymerase 
$(1 \mathrm{U} / \mathrm{mL})$, and $2 \mu \mathrm{L}$ of bacterial DNA. Amplification conditions were performed in a Thermocycler (Bio-Rad) following the above-mentioned protocol: one cycle at $94{ }^{\circ} \mathrm{C}$ for $5.5 \mathrm{~min}$, a first phase encompassing both start and denaturation cycles; 30 alternating cycles of annealing and extension for $30 \mathrm{~s}$ at $55^{\circ} \mathrm{C}$ and $90 \mathrm{~s}$ at $72{ }^{\circ} \mathrm{C}$, respectively; one final cycle at $72{ }^{\circ} \mathrm{C}$ for $90 \mathrm{~s}$ for the final extension. After the last cycle, the products were cooled to $12{ }^{\circ} \mathrm{C}$. PCR product purification was performed according to the GRS PCR and Gel Purification Kit (Grisp) protocol and used as templates. The resulting DNA sequences were aligned and compared in the Gene Bank through the use of the BLAST program (http:/ / www.ncbi.nlm.nih.gov, accessed on 20 June 2020) [30].

\subsection{Study of Antimicrobial Activity of Several Natural Products}

The antimicrobial activity of ten natural products was tested against each selected isolate, and the most effective natural compound was chosen for further experiments.

\subsubsection{Preparation of the Inoculum}

Inoculation of each isolate was performed from each culture grown on TSAYE or MRS (Biokar Diagnosis) at $30^{\circ} \mathrm{C}$ for $24 \mathrm{~h}$, by suspending isolated colonies in Ringer's solution to obtain a turbidity equivalent to $0.5 \mathrm{McF}$ arland scale.

\subsubsection{Preparation of Natural Products}

Ethanolic Propolis Extracts (EPE)

Two propolis samples from different geographic locations were used: one sample collected from Maia, Porto, Portugal (EPE1) and the other from Vila Franca, Viana do Castelo, Portugal (EPE2). Stock solutions of $0.1 \mathrm{~g} / \mathrm{mL}$ ethanolic propolis extracts were prepared for each sample, according to Casquete et al. [17]. Each propolis sample was extracted in $95 \%(v / v)$ ethanol (Sigma) for $24 \mathrm{~h}$ with magnetic agitation. Afterwards, the resulting mixture was filtered, and the residue was re-extracted in the same conditions. The filtrate solutions were combined, and each extract $(0.1 \mathrm{~g} / \mathrm{mL})$ was stored at room temperature in the dark.

\section{Chitosan Solutions}

Chitosans of different molecular weights were obtained from Sigma-Aldrich (St. Louis, USA): low molecular weight (LMW; deacetylation degree of 75-85\%), medium molecular weight (MMW; deacetylation degree of 75-85\%), and high molecular weight (HMW1; $>75 \%$ ). In addition, another chitosan of HMW was used (HMW2; Aqua Premier Co., Thailand; deacetylation degree of $90 \%)$. Chitosan solutions were prepared in a $3 \%(v / v)$ solution of glacial acetic acid 99\% (Panreac, Barcelona, Spain) to a final concentration of $30 \mathrm{mg} / \mathrm{mL}$, according to Casquete et al. [31]. Afterwards, the solution was stirred overnight for the dissolution of chitosan.

\section{Carvacrol and Limonene Solutions}

Solutions of $0.05 \%(v / v)$ carvacrol (99\%, Sigma-Aldrich) and 0.05\% (v/v) limonene ( $\geq 95 \%$, Sigma-Aldrich) were prepared by dissolution in polysorbate 80 (Sigma-Aldrich). The solutions were prepared just before usage.

\section{Olive Leaf Extract Solution}

A solution of $1.5 \%(w / v)$ olive leaf extract (Nutexa, Hoylake, UK) was prepared by dissolution of $0.3 \mathrm{~g}$ of the extract in $20 \mathrm{~mL}$ sterile distilled water. Since the active compound oleuropein is presented in $20 \%(w / w)$ of the olive leaf extract, only $0.3 \%(w / v)$ of oleuropein was present in the final solution.

\section{Citrox ${ }^{\circledR}$ Solution}

The Citrox ${ }^{\circledR}$ solution used was Citrox-ProGarda ${ }^{\mathrm{TM}}$ Fruit and Vegetable Decontaminant (ref. 14WP; Cirras Ltd., Middlesbrough, England), and it was diluted in sterile distilled water. 
Citrox-ProGarda ${ }^{\mathrm{TM}}$ Fruit and Vegetable Decontaminant is a natural, food-grade processing aid, free of genetically modified organisms, based on a citrus extract, with a combination of phenolic compounds and organic acids as its active ingredients. Dilutions were prepared in sterile water, and a solution of 50\% (v/v) was used in the antimicrobial assay.

\subsubsection{Antimicrobial Activity Screening of Each Natural Solution}

The antimicrobial activity of each solution was evaluated by the agar diffusion method [32]. Each solution was combined with the emulsifier polysorbate 80 in the proportion of 1:10 (polysorbate 80: natural compound solution). Blank disks (Oxoid, Basingstoke, United Kingdom) were immersed in each solution and left submerged for 30 min to absorb the solution. Sterile swabs were immersed in each inoculum suspension (prepared as described in Section 2.4.1) and spread in Müeller-Hinton Agar (MHA, Biokar diagnostics) plates. Disks containing the solutions were added to the top of the culture medium, and plates were incubated at $30^{\circ} \mathrm{C}$ for $24 \mathrm{~h}$. Disks adsorbed with distilled water, polysorbate 80 , acetic acid (PanReac), and ethanol (Sigma) were used as controls.

\subsubsection{Minimum Inhibitory and Bactericidal Concentrations of Effective Natural Compounds}

Minimum inhibitory (MIC) and bactericidal (MBC) concentrations were assessed only for Citrox ${ }^{\circledR}$ solution, which was the only product showing antimicrobial activity for the majority of the isolates. Following the protocol for microdilution broth susceptibility test described by Aumeeruddy-Elalfi et al. [33], $100 \mu \mathrm{L}$ of a solution containing $10 \mu \mathrm{L}$ of polysorbate 80 and $90 \mu \mathrm{L}$ of Müeller-Hinton Broth was distributed to each well of the 96-well microplates. The Citrox ${ }^{\circledR}$ solution was diluted, and eight different concentrations, ranging from $0.39 \%$ to $50 \%(v / v)$, were distributed throughout the wells. Then, $50 \mu \mathrm{L}$ of each inoculum, with a turbidity equivalent to $0.5 \mathrm{McF}$ arland scale, was added to each well, and the microplates were incubated at $30{ }^{\circ} \mathrm{C}$ for $24 \mathrm{~h}$. After the incubation period, $40 \mu \mathrm{L}$ of a $0.02 \%(w / v)$ solution of iodonitrotetrazolium (INT) chloride Biochema dye (PanReac) was added to each well. The reaction between INT chloride and viable bacterial cells in the suspension induced a change of color, from a transparent mild yellow to an intense red due to the reduction of INT chloride into INT formazan. The lowest concentration of Citrox ${ }^{\circledR}$ in which there was no change of color was considered the MIC.

To determine whether the compound was bactericidal or bacteriostatic, $10 \mu \mathrm{L}$ of suspension was collected from the wells not experiencing a color change in the microplate and inoculated on Müeller-Hinton Agar. The plates were incubated at $30^{\circ} \mathrm{C}$ for $24 \mathrm{~h}$ and checked for the presence of growth.

\section{Results}

Microbiological enumeration was performed for samples of two batches immediately after reception (0 days) and after 2 and 5 days of storage, according to each specific ISO standard and at $11^{\circ} \mathrm{C}$. The obtained results $(\log \mathrm{CFU} / \mathrm{g})$ are presented in Table 1.

Table 1. Microbial characterization of uncooked squid and shrimp skewers.

\begin{tabular}{|c|c|c|c|c|c|c|}
\hline & \multicolumn{6}{|c|}{$\log \mathrm{CFU} / \mathrm{g}$} \\
\hline & \multicolumn{3}{|c|}{ Standard Temperature } & \multicolumn{3}{|c|}{$11^{\circ} \mathrm{C}$} \\
\hline & Day 0 & Day 2 & Day 5 & Day 0 & Day 2 & Day 5 \\
\hline Listeria monocytogenes & $<1.00 \pm 0.00$ & $<1.00 \pm 0.00$ & $<1.00 \pm 0.00$ & $<1.00 \pm 0.00$ & $<1.00 \pm 0.00$ & $<1.00 \pm 0.00$ \\
\hline Escherichia coli & $<1.00 \pm 0.00$ & $<1.00 \pm 0.00$ & $<1.00 \pm 0.00$ & $<1.00 \pm 0.00$ & $<1.00 \pm 0.00$ & $<1.00 \pm 0.00$ \\
\hline Total viable counts & $5.49 \pm 0.54$ & $8.27 \pm 0.25$ & $9.46 \pm 0.17$ & $5.90 \pm 0.51$ & $8.64 \pm 0.91$ & $9.35 \pm 0.11$ \\
\hline Lactic Acid Bacteria & $3.74 \pm 0.15$ & $6.13 \pm 0.46$ & $7.24 \pm 0.10$ & $4.65 \pm<0.01$ & $6.84 \pm 0.18$ & $7.69 \pm 0.05$ \\
\hline Pseudomonas spp. & $6.21 \pm 0.20$ & $8.24 \pm 0.31$ & $9.20 \pm 0.11$ & $6.16 \pm 0.18$ & $8.42 \pm 0.03$ & $9.22 \pm 0.12$ \\
\hline Enterobacterales & $4.71 \pm 0.21$ & $7.18 \pm 0.8$ & $8.31 \pm 0.23$ & $4.74 \pm 0.01$ & $7.42 \pm 0.47$ & $8.16 \pm 0.02$ \\
\hline
\end{tabular}


Isolated colonies from samples enumerated immediately after the reception were identified through 16s rRNA sequencing. In Tables 2 and 3 are shown the medium from which these isolates were recovered and the several species identified, respectively.

Table 2. Number of isolates collected per culture medium.

\begin{tabular}{|c|c|c|c|c|}
\hline & PCA & MRS & РAB & RAPID' Enterobacteriaceae \\
\hline Number of isolates & 39 & 22 & 13 & 26 \\
\hline
\end{tabular}

Table 3. Identification of 100 isolates by $16 \mathrm{~S}$ rRNA sequencing.

\begin{tabular}{|c|c|c|}
\hline Identification & Number of Isolates & 16S rRNA Sequencing Similarity $(\%)$ \\
\hline Hafnia alvei & 6 & $99.40-99.90$ \\
\hline Klebsiella oxytoca & 2 & $99.90-100.00$ \\
\hline Latilactobacillus sakei & 5 & $99.90-100.00$ \\
\hline Lactococcus garvieae & 2 & $99.90-100.00$ \\
\hline Lactococcus piscium & 1 & $99.40-99.90$ \\
\hline Leuconostoc gelidum & 1 & $99.40-99.90$ \\
\hline Leuconostoc mesenteroides & 1 & 100.00 \\
\hline Leuconostoc miyukkimchii & 12 & $99.10-99.90$ \\
\hline Morganella morganii & 3 & 99.80 \\
\hline Obesumbacterium proteus & 1 & 99.90 \\
\hline Pantoea brenneri & 1 & 98.50 \\
\hline Pseudomonas endophytica & 1 & 99.70 \\
\hline Pseudomonas fragi & 2 & 99.70 \\
\hline Pseudomonas helleri & 3 & $99.80-100.00$ \\
\hline Pseudomonas helmanticensis & 1 & 99.80 \\
\hline Pseudomonas marginalis & 1 & 100.00 \\
\hline Pseudomonas orientalis & 1 & 99.80 \\
\hline Pseudomonas paralactis & 6 & $99.80-100.00$ \\
\hline Pseudomonas psychrophila & 3 & $99.70-99.90$ \\
\hline Pseudomonas trivialis & 4 & 99.90 \\
\hline Pseudomonas weihenstephanensis & 2 & $99.60-99.90$ \\
\hline Rahnella aquatilis & 4 & $99.60-99.90$ \\
\hline Rahnella inusitata & 10 & $99.80-99.90$ \\
\hline Serratia fonticola & 2 & 99.90 \\
\hline Serratia liquefaciens & 20 & $99.40-99.90$ \\
\hline Serratia nematodiphila & 1 & 100.00 \\
\hline Weissella fabalis & 3 & $97.10-98.10$ \\
\hline Weissella beninensis & 1 & 97.60 \\
\hline
\end{tabular}

The results of the antimicrobial activity assay with the respective diameter of inhibition halos observed for each compound against each identified isolate are presented in Table 4. 
Table 4. Diameters of inhibition halos ( $\mathrm{mm}$ ) and susceptibility obtained for each compound against each identified isolate.

\begin{tabular}{|c|c|c|c|c|c|c|c|c|c|c|c|c|c|}
\hline Car. & $\mathrm{T} 80$ & OLE & Lim. & C. HMW1 & C. HMW2 & C. MMW & C. LMW & A.A. & EEP 1 & EEP 2 & ETOH & Citrox $^{\circledR}$ & Identified Isolate \\
\hline $0(\mathrm{R})$ & $0(\mathrm{R})$ & $0(\mathrm{R})$ & $0(\mathrm{R})$ & $14(\mathrm{~S})$ & $15(\mathrm{~S})$ & $15(\mathrm{~S})$ & $14(\mathrm{~S})$ & $16(\mathrm{~S})$ & $0(\mathrm{R})$ & $0(\mathrm{R})$ & $0(\mathrm{R})$ & $17(\mathrm{~S})$ & Pseudomonas endophytica \\
\hline $0(\mathrm{R})$ & $0(\mathrm{R})$ & $0(\mathrm{R})$ & $0(\mathrm{R})$ & $14(\mathrm{~S})$ & $12(\mathrm{~S})$ & $11(\mathrm{~S})$ & 15 (S) & 16 (S) & $7(\mathrm{R})$ & $8(\mathrm{R})$ & $0(\mathrm{R})$ & $21(\mathrm{~S})$ & Pseudomonas fragi \\
\hline 0 (R) & $0(\mathrm{R})$ & 0 (R) & $0(\mathrm{R})$ & $14(\mathrm{~S})$ & $12(\mathrm{~S})$ & 11 (S) & 15 (S) & 16 (S) & $7(\mathrm{R})$ & $8(\mathrm{R})$ & 0 (R) & $21(\mathrm{~S})$ & Pseudomonas fragi \\
\hline $0(\mathrm{R})$ & $0(\mathrm{R})$ & $0(\mathrm{R})$ & $0(\mathrm{R})$ & 14 (S) & $14(\mathrm{~S})$ & $14(\mathrm{~S})$ & 14 (S) & 14 (S) & $7(\mathrm{R})$ & $0(\mathrm{R})$ & $0(\mathrm{R})$ & $23(\mathrm{~S})$ & Pseudomonas helleri \\
\hline 0 (R) & $0(\mathrm{R})$ & 0 (R) & 0 (R) & $21(\mathrm{~S})$ & $24(\mathrm{~S})$ & $27(\mathrm{~S})$ & $27(\mathrm{~S})$ & 24 (S) & $9(\mathrm{R})$ & $8(\mathrm{R})$ & $0(\mathrm{R})$ & $31(\mathrm{~S})$ & Pseudomonas helleri \\
\hline $0(\mathrm{R})$ & $0(\mathrm{R})$ & 0 (R) & $0(\mathrm{R})$ & 15 (S) & $15(\mathrm{~S})$ & $16(\mathrm{~S})$ & $16(\mathrm{~S})$ & 17 (S) & $0(\mathrm{R})$ & $8(\mathrm{R})$ & $0(\mathrm{R})$ & $24(\mathrm{~S})$ & Pseudomonas helleri \\
\hline $0(\mathrm{R})$ & $0(\mathrm{R})$ & 0 (R) & $0(\mathrm{R})$ & 14 (S) & 15 (S) & $14(\mathrm{~S})$ & 16 (S) & 15 (S) & $9(\mathrm{R})$ & $0(\mathrm{R})$ & $0(\mathrm{R})$ & 24 (S) & Pseudomonas helmanticensis \\
\hline $0(\mathrm{R})$ & $0(\mathrm{R})$ & $0(\mathrm{R})$ & $0(\mathrm{R})$ & 16 (S) & 16 (S) & 14 (S) & 16 (S) & 20 (S) & $0(\mathrm{R})$ & $9(\mathrm{R})$ & $0(\mathrm{R})$ & 31 (S) & Pseudomonas marginalis \\
\hline $0(\mathrm{R})$ & $0(\mathrm{R})$ & $0(\mathrm{R})$ & $0(\mathrm{R})$ & $14(\mathrm{~S})$ & 15 (S) & 14 (S) & 15 (S) & 14 (S) & $0(\mathrm{R})$ & $0(\mathrm{R})$ & $0(\mathrm{R})$ & 25 (S) & Pseudomonas orientalis \\
\hline $0(\mathrm{R})$ & $0(\mathrm{R})$ & $0(\mathrm{R})$ & $0(\mathrm{R})$ & $16(\mathrm{~S})$ & $17(\mathrm{~S})$ & 17 (S) & 15 (S) & 15 (S) & $7(\mathrm{R})$ & $8(\mathrm{R})$ & $0(\mathrm{R})$ & $22(\mathrm{~S})$ & Pseudomonas paralactis \\
\hline 0 (R) & 0 (R) & 0 (R) & 0 (R) & 14 (S) & $15(\mathrm{~S})$ & $13(\mathrm{~S})$ & 15 (S) & 13 (S) & $0(\mathrm{R})$ & $7(\mathrm{R})$ & 0 (R) & 25 (S) & Pseudomonas paralactis \\
\hline $0(\mathrm{R})$ & $0(\mathrm{R})$ & 0 (R) & 0 (R) & 20 (S) & $21(\mathrm{~S})$ & $21(\mathrm{~S})$ & $22(\mathrm{~S})$ & 23 (S) & $0(\mathrm{R})$ & $8(\mathrm{R})$ & $0(\mathrm{R})$ & 30 (S) & Pseudomonas paralactis \\
\hline 0 (R) & $0(\mathrm{R})$ & $0(\mathrm{R})$ & $0(\mathrm{R})$ & 17 (S) & 20 (S) & 17 (S) & 20 (S) & 20 (S) & $9(\mathrm{R})$ & $0(\mathrm{R})$ & $0(\mathrm{R})$ & 30 (S) & Pseudomonas paralactis \\
\hline $0(\mathrm{R})$ & $0(\mathrm{R})$ & 0 (R) & $0(\mathrm{R})$ & 14 (S) & 15 (S) & $14(\mathrm{~S})$ & 16 (S) & 18 (S) & $8(\mathrm{R})$ & $8(\mathrm{R})$ & $7(\mathrm{R})$ & 30 (S) & Pseudomonas paralactis \\
\hline $0(\mathrm{R})$ & $0(\mathrm{R})$ & $0(\mathrm{R})$ & $0(\mathrm{R})$ & $18(\mathrm{~S})$ & 15 (S) & $20(S)$ & 15 (S) & 16 (S) & $7(\mathrm{R})$ & $8(\mathrm{R})$ & $0(\mathrm{R})$ & $22(\mathrm{~S})$ & Pseudomonas psychrophila \\
\hline $0(\mathrm{R})$ & $0(\mathrm{R})$ & $0(\mathrm{R})$ & $0(\mathrm{R})$ & $13(\mathrm{~S})$ & $14(\mathrm{~S})$ & 16 (S) & $15(\mathrm{~S})$ & 14 (S) & $7(\mathrm{R})$ & $8(\mathrm{R})$ & $0(\mathrm{R})$ & $22(\mathrm{~S})$ & Pseudomonas psychrophila \\
\hline $0(\mathrm{R})$ & $0(\mathrm{R})$ & $0(\mathrm{R})$ & $0(\mathrm{R})$ & $12(\mathrm{~S})$ & $17(\mathrm{~S})$ & $15(\mathrm{~S})$ & 18 (S) & 16 (S) & $9(\mathrm{R})$ & $8(\mathrm{R})$ & $0(\mathrm{R})$ & 24 (S) & Pseudomonas psychrophila \\
\hline 0 (R) & $0(\mathrm{R})$ & 0 (R) & 0 (R) & 10 (S) & $13(\mathrm{~S})$ & $10(\mathrm{~S})$ & $12(\mathrm{~S})$ & 11 (S) & $7(\mathrm{R})$ & $8(\mathrm{R})$ & 0 (R) & $23(\mathrm{~S})$ & Pseudomonas trivialis \\
\hline $0(\mathrm{R})$ & $0(\mathrm{R})$ & 0 (R) & $0(\mathrm{R})$ & $16(\mathrm{~S})$ & $17(\mathrm{~S})$ & $15(\mathrm{~S})$ & $17(\mathrm{~S})$ & 17 (S) & $0(\mathrm{R})$ & $0(\mathrm{R})$ & $0(\mathrm{R})$ & $23(\mathrm{~S})$ & Pseudomonas trivialis \\
\hline $0(\mathrm{R})$ & $0(\mathrm{R})$ & $0(\mathrm{R})$ & $0(\mathrm{R})$ & 14 (S) & 15 (S) & $13(\mathrm{~S})$ & 15 (S) & 18 (S) & $0(\mathrm{R})$ & $8(\mathrm{R})$ & $0(\mathrm{R})$ & 25 (S) & Pseudomonas trivialis \\
\hline $0(\mathrm{R})$ & $0(\mathrm{R})$ & $0(\mathrm{R})$ & $0(\mathrm{R})$ & 19 (S) & 20 (S) & 17 (S) & $21(\mathrm{~S})$ & 21 (S) & $0(\mathrm{R})$ & $0(\mathrm{R})$ & $0(\mathrm{R})$ & 24 (S) & Pseudomonas weihenstephanensis \\
\hline $0(\mathrm{R})$ & $0(\mathrm{R})$ & $0(\mathrm{R})$ & $0(\mathrm{R})$ & $11(\mathrm{~S})$ & $11(\mathrm{~S})$ & $11(\mathrm{~S})$ & $11(\mathrm{~S})$ & 10 (S) & $0(\mathrm{R})$ & $0(\mathrm{R})$ & $0(\mathrm{R})$ & 16 (S) & Hafnia alvei \\
\hline $0(\mathrm{R})$ & $0(\mathrm{R})$ & 0 (R) & 0 (R) & 11 (S) & $12(\mathrm{~S})$ & $10(\mathrm{~S})$ & $10(\mathrm{~S})$ & 11 (S) & $0(\mathrm{R})$ & $8(\mathrm{R})$ & $0(\mathrm{R})$ & $21(\mathrm{~S})$ & Hafnia alvei \\
\hline $0(\mathrm{R})$ & $0(\mathrm{R})$ & 0 (R) & $0(\mathrm{R})$ & $13(\mathrm{~S})$ & $12(\mathrm{~S})$ & 11 (S) & $12(\mathrm{~S})$ & $13(\mathrm{~S})$ & $0(\mathrm{R})$ & $0(\mathrm{R})$ & $0(\mathrm{R})$ & 15 (S) & Hafnia alvei \\
\hline $0(\mathrm{R})$ & $0(\mathrm{R})$ & $0(\mathrm{R})$ & $0(\mathrm{R})$ & 13 (S) & $12(\mathrm{~S})$ & 11 (S) & $12(\mathrm{~S})$ & 13 (S) & $0(\mathrm{R})$ & $0(\mathrm{R})$ & $0(\mathrm{R})$ & 15 (S) & Hafnia alvei \\
\hline $0(\mathrm{R})$ & $0(\mathrm{R})$ & $0(\mathrm{R})$ & $0(\mathrm{R})$ & 10 (S) & 10 (S) & $9(\mathrm{R})$ & $10(\mathrm{~S})$ & 12 (S) & $8(\mathrm{R})$ & $0(\mathrm{R})$ & $7(\mathrm{R})$ & 20 (S) & Hafnia alvei \\
\hline $0(\mathrm{R})$ & $0(\mathrm{R})$ & $0(\mathrm{R})$ & $0(\mathrm{R})$ & $10(\mathrm{~S})$ & $13(\mathrm{~S})$ & $13(\mathrm{~S})$ & $12(\mathrm{~S})$ & 13 (S) & $0(\mathrm{R})$ & $8(\mathrm{R})$ & $0(\mathrm{R})$ & 18 (S) & Hafnia alvei \\
\hline 0 (R) & $0(\mathrm{R})$ & $0(\mathrm{R})$ & $0(\mathrm{R})$ & $11(\mathrm{~S})$ & $12(\mathrm{~S})$ & $10(S)$ & $12(\mathrm{~S})$ & $13(\mathrm{~S})$ & $0(\mathrm{R})$ & $7(\mathrm{R})$ & 0 (R) & $19(\mathrm{~S})$ & Klebsiella oxytoca \\
\hline $0(\mathrm{R})$ & $0(\mathrm{R})$ & $0(\mathrm{R})$ & $0(\mathrm{R})$ & $12(\mathrm{~S})$ & $14(\mathrm{~S})$ & $13(\mathrm{~S})$ & 14 (S) & 15 (S) & $0(\mathrm{R})$ & $8(\mathrm{R})$ & $0(\mathrm{R})$ & 16 (S) & Klebsiella oxytoca \\
\hline 0 (R) & $0(\mathrm{R})$ & 0 (R) & 0 (R) & 15 (S) & $15(\mathrm{~S})$ & $13(\mathrm{~S})$ & $15(\mathrm{~S})$ & 16 (S) & $8(\mathrm{R})$ & $0(\mathrm{R})$ & $0(\mathrm{R})$ & $23(\mathrm{~S})$ & Morganella morganii \\
\hline $0(\mathrm{R})$ & $0(\mathrm{R})$ & 0 (R) & 0 (R) & $16(\mathrm{~S})$ & $14(\mathrm{~S})$ & $14(\mathrm{~S})$ & $14(\mathrm{~S})$ & 15 (S) & $0(\mathrm{R})$ & $7(\mathrm{R})$ & $0(\mathrm{R})$ & 25 (S) & Morganella morganii \\
\hline 0 (R) & $0(\mathrm{R})$ & 0 (R) & $0(\mathrm{R})$ & 10 (S) & $10(\mathrm{~S})$ & $10(\mathrm{~S})$ & 10 (S) & 10 (S) & $8(\mathrm{R})$ & $8(\mathrm{R})$ & $0(\mathrm{R})$ & $22(\mathrm{~S})$ & Morganella morganii \\
\hline $0(\mathrm{R})$ & $0(\mathrm{R})$ & $0(\mathrm{R})$ & $0(\mathrm{R})$ & $8(\mathrm{R})$ & $8(\mathrm{R})$ & $8(\mathrm{R})$ & $9(\mathrm{R})$ & $7(\mathrm{R})$ & $8(\mathrm{R})$ & $7(\mathrm{R})$ & $0(\mathrm{R})$ & 14 (S) & Obesumbacterium proteus \\
\hline $0(\mathrm{R})$ & $0(\mathrm{R})$ & $0(\mathrm{R})$ & $0(\mathrm{R})$ & $11(\mathrm{~S})$ & $10(\mathrm{~S})$ & $11(\mathrm{~S})$ & $11(\mathrm{~S})$ & $12(\mathrm{~S})$ & $7(\mathrm{R})$ & $7(\mathrm{R})$ & $0(\mathrm{R})$ & 15 (S) & Pantoea brenneri \\
\hline $0(\mathrm{R})$ & $0(\mathrm{R})$ & 0 (R) & $0(\mathrm{R})$ & $17(\mathrm{~S})$ & $16(\mathrm{~S})$ & $17(\mathrm{~S})$ & 16 (S) & 18 (S) & $8(\mathrm{R})$ & $9(\mathrm{R})$ & 0 (R) & $23(\mathrm{~S})$ & Rahnella aquatilis \\
\hline $0(\mathrm{R})$ & $0(\mathrm{R})$ & $0(\mathrm{R})$ & $0(\mathrm{R})$ & $17(\mathrm{~S})$ & 18 (S) & $16(\mathrm{~S})$ & $17(\mathrm{~S})$ & 18 (S) & $0(\mathrm{R})$ & $0(\mathrm{R})$ & $0(\mathrm{R})$ & $21(\mathrm{~S})$ & Rahnella aquatilis \\
\hline 0 (R) & $0(\mathrm{R})$ & 0 (R) & 0 (R) & 16 (S) & $18(\mathrm{~S})$ & $15(\mathrm{~S})$ & $16(\mathrm{~S})$ & 15 (S) & $0(\mathrm{R})$ & $8(\mathrm{R})$ & $0(\mathrm{R})$ & 16 (S) & Rahnella aquatilis \\
\hline $0(\mathrm{R})$ & $0(\mathrm{R})$ & $0(\mathrm{R})$ & $0(\mathrm{R})$ & $14(\mathrm{~S})$ & $15(\mathrm{~S})$ & $14(\mathrm{~S})$ & $17(\mathrm{~S})$ & 18 (S) & $0(\mathrm{R})$ & $7(\mathrm{R})$ & $0(\mathrm{R})$ & $23(\mathrm{~S})$ & Rahnella aquatilis \\
\hline $0(\mathrm{R})$ & $0(\mathrm{R})$ & $0(\mathrm{R})$ & $0(\mathrm{R})$ & $0(\mathrm{R})$ & $0(\mathrm{R})$ & $0(\mathrm{R})$ & $0(\mathrm{R})$ & $0(\mathrm{R})$ & $0(\mathrm{R})$ & $0(\mathrm{R})$ & $0(\mathrm{R})$ & 15 (S) & Rahnella inusitata \\
\hline
\end{tabular}


Table 4. Cont.

\begin{tabular}{|c|c|c|c|c|c|c|c|c|c|c|c|c|c|}
\hline Car. & T80 & OLE & Lim. & C. HMW1 & C. HMW2 & C. MMW & C. LMW & A.A. & EEP 1 & EEP 2 & ETOH & Citrox $^{\circledR}$ & Identified Isolate \\
\hline $0(\mathrm{R})$ & $0(\mathrm{R})$ & $0(\mathrm{R})$ & $0(\mathrm{R})$ & $13(S)$ & $13(\mathrm{~S})$ & $10(\mathrm{~S})$ & $10(\mathrm{~S})$ & $11(\mathrm{~S})$ & $7(\mathrm{R})$ & $9(\mathrm{R})$ & $0(\mathrm{R})$ & $19(\mathrm{~S})$ & Rahnella inusitata \\
\hline $0(\mathrm{R})$ & $0(\mathrm{R})$ & $0(\mathrm{R})$ & $0(\mathrm{R})$ & $12(\mathrm{~S})$ & $12(\mathrm{~S})$ & $13(\mathrm{~S})$ & $11(\mathrm{~S})$ & 11 (S) & $8(\mathrm{R})$ & $7(\mathrm{R})$ & $0(\mathrm{R})$ & $18(\mathrm{~S})$ & Rahnella inusitata \\
\hline 0 (R) & $0(\mathrm{R})$ & $0(\mathrm{R})$ & 0 (R) & $12(\mathrm{~S})$ & $12(\mathrm{~S})$ & $12(\mathrm{~S})$ & $15(\mathrm{~S})$ & 13 (S) & 7 (R) & $0(\mathrm{R})$ & $0(\mathrm{R})$ & $17(\mathrm{~S})$ & Rahnella inusitata \\
\hline $0(\mathrm{R})$ & $0(\mathrm{R})$ & $0(\mathrm{R})$ & 0 (R) & $14(\mathrm{~S})$ & $13(\mathrm{~S})$ & 11 (S) & $13(\mathrm{~S})$ & 13 (S) & $0(\mathrm{R})$ & $7(\mathrm{R})$ & $0(\mathrm{R})$ & $33(\mathrm{~S})$ & Rahnella inusitata \\
\hline 0 (R) & $0(\mathrm{R})$ & $0(\mathrm{R})$ & $0(\mathrm{R})$ & $11(\mathrm{~S})$ & $12(\mathrm{~S})$ & $11(\mathrm{~S})$ & $12(\mathrm{~S})$ & $13(\mathrm{~S})$ & $0(\mathrm{R})$ & $8(\mathrm{R})$ & 7 (R) & 19 (S) & Rahnella inusitata \\
\hline 0 (R) & $0(\mathrm{R})$ & $0(\mathrm{R})$ & 0 (R) & $16(\mathrm{~S})$ & $17(\mathrm{~S})$ & $16(\mathrm{~S})$ & $16(\mathrm{~S})$ & 17 (S) & $0(\mathrm{R})$ & $0(\mathrm{R})$ & $0(\mathrm{R})$ & $25(\mathrm{~S})$ & Rahnella inusitata \\
\hline 0 (R) & $0(\mathrm{R})$ & $0(\mathrm{R})$ & $0(\mathrm{R})$ & $17(\mathrm{~S})$ & $19(\mathrm{~S})$ & $19(\mathrm{~S})$ & $20(\mathrm{~S})$ & 19 (S) & $9(\mathrm{R})$ & $0(\mathrm{R})$ & $0(\mathrm{R})$ & $22(\mathrm{~S})$ & Rahnella inusitata \\
\hline $0(\mathrm{R})$ & $0(\mathrm{R})$ & $0(\mathrm{R})$ & $0(\mathrm{R})$ & $15(\mathrm{~S})$ & $15(\mathrm{~S})$ & $14(\mathrm{~S})$ & $14(\mathrm{~S})$ & 16 (S) & $0(\mathrm{R})$ & $7(\mathrm{R})$ & 0 (R) & $22(\mathrm{~S})$ & Rahnella inusitata \\
\hline 0 (R) & $0(\mathrm{R})$ & $0(\mathrm{R})$ & 0 (R) & $11(\mathrm{~S})$ & $14(\mathrm{~S})$ & $12(\mathrm{~S})$ & $14(\mathrm{~S})$ & 14 (S) & 7 (R) & $7(\mathrm{R})$ & $0(\mathrm{R})$ & $18(\mathrm{~S})$ & Serratia fonticola \\
\hline $8(\mathrm{R})$ & $10(\mathrm{~S})$ & $0(\mathrm{R})$ & $9(\mathrm{R})$ & $15(\mathrm{~S})$ & $16(\mathrm{~S})$ & $14(\mathrm{~S})$ & $17(\mathrm{~S})$ & $15(\mathrm{~S})$ & $0(\mathrm{R})$ & $0(\mathrm{R})$ & $0(\mathrm{R})$ & 19 (S) & Serratia liquefaciens \\
\hline $8(\mathrm{R})$ & 11 (S) & $0(\mathrm{R})$ & $10(\mathrm{~S})$ & $11(\mathrm{~S})$ & $11(\mathrm{~S})$ & $10(\mathrm{~S})$ & $9(\mathrm{R})$ & 10 (S) & 7 (R) & $8(\mathrm{R})$ & $8(\mathrm{R})$ & 17 (S) & Serratia liquefaciens \\
\hline 0 (R) & $0(\mathrm{R})$ & $0(\mathrm{R})$ & $0(\mathrm{R})$ & $11(\mathrm{~S})$ & $8(\mathrm{R})$ & $10(\mathrm{~S})$ & 8 (R) & 10 (S) & $7(\mathrm{R})$ & $0(\mathrm{R})$ & $0(\mathrm{R})$ & 19 (S) & Serratia liquefaciens \\
\hline $7(\mathrm{R})$ & $9(\mathrm{R})$ & $0(\mathrm{R})$ & $9(\mathrm{R})$ & $14(\mathrm{~S})$ & $15(\mathrm{~S})$ & $12(\mathrm{~S})$ & $14(\mathrm{~S})$ & $15(\mathrm{~S})$ & $8(\mathrm{R})$ & $8(\mathrm{R})$ & $13(\mathrm{~S})$ & $18(\mathrm{~S})$ & Serratia liquefaciens \\
\hline $0(\mathrm{R})$ & $0(\mathrm{R})$ & $0(\mathrm{R})$ & $0(\mathrm{R})$ & $8(\mathrm{R})$ & $9(\mathrm{R})$ & $7(\mathrm{R})$ & $9(\mathrm{R})$ & $8(\mathrm{R})$ & $0(\mathrm{R})$ & $7(\mathrm{R})$ & $0(\mathrm{R})$ & $18(\mathrm{~S})$ & Serratia liquefaciens \\
\hline 0 (R) & $0(\mathrm{R})$ & $0(\mathrm{R})$ & 0 (R) & $12(\mathrm{~S})$ & $12(\mathrm{~S})$ & $10(\mathrm{~S})$ & $12(\mathrm{~S})$ & 15 (S) & $8(\mathrm{R})$ & $0(\mathrm{R})$ & $0(\mathrm{R})$ & 16 (S) & Serratia liquefaciens \\
\hline $8(\mathrm{R})$ & $8(\mathrm{R})$ & $0(\mathrm{R})$ & $9(\mathrm{R})$ & $12(S)$ & $15(\mathrm{~S})$ & $13(\mathrm{~S})$ & $13(S)$ & 14 (S) & $8(\mathrm{R})$ & $7(\mathrm{R})$ & $0(\mathrm{R})$ & $12(\mathrm{~S})$ & Serratia liquefaciens \\
\hline $9(\mathrm{R})$ & $10(\mathrm{~S})$ & $0(\mathrm{R})$ & $10(\mathrm{~S})$ & $15(\mathrm{~S})$ & $15(\mathrm{~S})$ & 15 (S) & $13(\mathrm{~S})$ & $15(\mathrm{~S})$ & $7(\mathrm{R})$ & $8(\mathrm{R})$ & $0(\mathrm{R})$ & $18(\mathrm{~S})$ & Serratia liquefaciens \\
\hline $9(\mathrm{R})$ & $9(\mathrm{R})$ & $0(\mathrm{R})$ & 10 (S) & $15(\mathrm{~S})$ & $13(\mathrm{~S})$ & $14(\mathrm{~S})$ & $14(\mathrm{~S})$ & 14 (S) & $8(\mathrm{R})$ & $7(\mathrm{R})$ & $0(\mathrm{R})$ & 19 (S) & Serratia liquefaciens \\
\hline $9(\mathrm{R})$ & 11 (S) & $0(\mathrm{R})$ & $10(\mathrm{~S})$ & $10(\mathrm{~S})$ & $8(\mathrm{R})$ & $10(\mathrm{~S})$ & $10(\mathrm{~S})$ & $7(\mathrm{R})$ & $8(\mathrm{R})$ & $7(\mathrm{R})$ & $0(\mathrm{R})$ & 18 (S) & Serratia liquefaciens \\
\hline 0 (R) & $0(\mathrm{R})$ & $0(\mathrm{R})$ & $0(\mathrm{R})$ & $16(S)$ & $14(\mathrm{~S})$ & $12(\mathrm{~S})$ & $18(S)$ & 16 (S) & $0(\mathrm{R})$ & $8(\mathrm{R})$ & $0(\mathrm{R})$ & $19(\mathrm{~S})$ & Serratia liquefaciens \\
\hline $8(\mathrm{R})$ & $8(\mathrm{R})$ & $0(\mathrm{R})$ & $8(\mathrm{R})$ & $0(\mathrm{R})$ & $12(\mathrm{~S})$ & $12(\mathrm{~S})$ & $12(S)$ & 12 (S) & $0(\mathrm{R})$ & $8(\mathrm{R})$ & 0 (R) & $17(\mathrm{~S})$ & Serratia liquefaciens \\
\hline 0 (R) & $0(\mathrm{R})$ & $0(\mathrm{R})$ & $0(\mathrm{R})$ & $20(\mathrm{~S})$ & $20(\mathrm{~S})$ & 20 (S) & $20(S)$ & $24(\mathrm{~S})$ & $0(\mathrm{R})$ & $0(\mathrm{R})$ & $7(\mathrm{R})$ & $20(S)$ & Serratia liquefaciens \\
\hline $9(\mathrm{R})$ & $10(\mathrm{~S})$ & $0(\mathrm{R})$ & $10(\mathrm{~S})$ & $24(S)$ & $22(\mathrm{~S})$ & $16(\mathrm{~S})$ & $20(S)$ & 20 (S) & $0(\mathrm{R})$ & $0(\mathrm{R})$ & $0(\mathrm{R})$ & $20(S)$ & Serratia liquefaciens \\
\hline 0 (R) & $0(\mathrm{R})$ & $0(\mathrm{R})$ & $0(\mathrm{R})$ & $21(\mathrm{~S})$ & $19(\mathrm{~S})$ & $19(\mathrm{~S})$ & $19(\mathrm{~S})$ & 24 (S) & $9(\mathrm{R})$ & $8(\mathrm{R})$ & $0(\mathrm{R})$ & $22(\mathrm{~S})$ & Serratia liquefaciens \\
\hline $0(\mathrm{R})$ & $0(\mathrm{R})$ & $0(\mathrm{R})$ & $0(\mathrm{R})$ & $14(\mathrm{~S})$ & $14(\mathrm{~S})$ & $13(\mathrm{~S})$ & $15(\mathrm{~S})$ & $13(\mathrm{~S})$ & $0(\mathrm{R})$ & $7(\mathrm{R})$ & $0(\mathrm{R})$ & $19(\mathrm{~S})$ & Serratia liquefaciens \\
\hline $0(\mathrm{R})$ & $0(\mathrm{R})$ & $0(\mathrm{R})$ & $0(\mathrm{R})$ & $11(\mathrm{~S})$ & $13(\mathrm{~S})$ & $15(\mathrm{~S})$ & $14(\mathrm{~S})$ & 16 (S) & $7(\mathrm{R})$ & $0(\mathrm{R})$ & $0(\mathrm{R})$ & $20(\mathrm{~S})$ & Serratia liquefaciens \\
\hline $0(\mathrm{R})$ & $0(\mathrm{R})$ & $0(\mathrm{R})$ & 0 (R) & $0(\mathrm{R})$ & $0(\mathrm{R})$ & $0(\mathrm{R})$ & $0(\mathrm{R})$ & $0(\mathrm{R})$ & $0(\mathrm{R})$ & $0(\mathrm{R})$ & $0(\mathrm{R})$ & $18(\mathrm{~S})$ & Serratia liquefaciens \\
\hline $7(\mathrm{R})$ & $9(\mathrm{R})$ & $0(\mathrm{R})$ & 7 (R) & 8 (R) & $13(\mathrm{~S})$ & $11(\mathrm{~S})$ & $12(\mathrm{~S})$ & $12(\mathrm{~S})$ & $0(\mathrm{R})$ & $0(\mathrm{R})$ & $0(\mathrm{R})$ & $17(\mathrm{~S})$ & Serratia nematodiphila \\
\hline $0(\mathrm{R})$ & $0(\mathrm{R})$ & $0(\mathrm{R})$ & $0(\mathrm{R})$ & $0(\mathrm{R})$ & $0(\mathrm{R})$ & $0(\mathrm{R})$ & $0(\mathrm{R})$ & $0(\mathrm{R})$ & $0(\mathrm{R})$ & $0(\mathrm{R})$ & $0(\mathrm{R})$ & $17(\mathrm{~S})$ & Latilactobacillus sakei \\
\hline $0(\mathrm{R})$ & $0(\mathrm{R})$ & $0(\mathrm{R})$ & $0(\mathrm{R})$ & $0(\mathrm{R})$ & $0(\mathrm{R})$ & $0(\mathrm{R})$ & $0(\mathrm{R})$ & $0(\mathrm{R})$ & $0(\mathrm{R})$ & $0(\mathrm{R})$ & $0(\mathrm{R})$ & $15(\mathrm{~S})$ & Latilactobacillus sakei \\
\hline 0 (R) & $0(\mathrm{R})$ & $0(\mathrm{R})$ & 0 (R) & $0(\mathrm{R})$ & 0 (R) & 0 (R) & $0(\mathrm{R})$ & $0(\mathrm{R})$ & $0(\mathrm{R})$ & $11(\mathrm{~S})$ & $0(\mathrm{R})$ & $19(\mathrm{~S})$ & Latilactobacillus sakei \\
\hline 0 (R) & $0(\mathrm{R})$ & $0(\mathrm{R})$ & $0(\mathrm{R})$ & $0(\mathrm{R})$ & $0(\mathrm{R})$ & $0(\mathrm{R})$ & $0(\mathrm{R})$ & $10(\mathrm{~S})$ & $12(\mathrm{~S})$ & $12(\mathrm{~S})$ & $0(\mathrm{R})$ & $30(\mathrm{~S})$ & Latilactobacillus sakei \\
\hline $0(\mathrm{R})$ & $0(\mathrm{R})$ & $0(\mathrm{R})$ & $0(\mathrm{R})$ & $0(\mathrm{R})$ & $0(\mathrm{R})$ & $0(\mathrm{R})$ & $0(\mathrm{R})$ & $0(\mathrm{R})$ & $0(\mathrm{R})$ & $0(\mathrm{R})$ & $0(\mathrm{R})$ & $13(\mathrm{~S})$ & Latilactobacillus sakei \\
\hline $0(\mathrm{R})$ & $0(\mathrm{R})$ & $0(\mathrm{R})$ & 0 (R) & $0(\mathrm{R})$ & $0(\mathrm{R})$ & $0(\mathrm{R})$ & $0(\mathrm{R})$ & 10 (S) & $11(\mathrm{~S})$ & $10(\mathrm{~S})$ & $0(\mathrm{R})$ & $30(\mathrm{~S})$ & Lactococcus garvieae \\
\hline $0(\mathrm{R})$ & $0(\mathrm{R})$ & $0(\mathrm{R})$ & $0(\mathrm{R})$ & $0(\mathrm{R})$ & $0(\mathrm{R})$ & $0(\mathrm{R})$ & $0(\mathrm{R})$ & $0(\mathrm{R})$ & $12(\mathrm{~S})$ & $10(\mathrm{~S})$ & $0(\mathrm{R})$ & $32(\mathrm{~S})$ & Lactococcus garvieae \\
\hline $0(\mathrm{R})$ & $0(\mathrm{R})$ & $0(\mathrm{R})$ & $0(\mathrm{R})$ & $18(\mathrm{~S})$ & 17 (S) & $15(\mathrm{~S})$ & $16(S)$ & $18(S)$ & $7(\mathrm{R})$ & $8(\mathrm{R})$ & $0(\mathrm{R})$ & $21(\mathrm{~S})$ & Lactococcus piscium \\
\hline $0(\mathrm{R})$ & $0(\mathrm{R})$ & $0(\mathrm{R})$ & $0(\mathrm{R})$ & $0(\mathrm{R})$ & $0(\mathrm{R})$ & $0(\mathrm{R})$ & $0(\mathrm{R})$ & $0(\mathrm{R})$ & $0(\mathrm{R})$ & $10(\mathrm{~S})$ & $0(\mathrm{R})$ & $15(\mathrm{~S})$ & Leuconostoc gelidum \\
\hline 0 (R) & $0(\mathrm{R})$ & $0(\mathrm{R})$ & 0 (R) & $0(\mathrm{R})$ & 0 (R) & $0(\mathrm{R})$ & $0(\mathrm{R})$ & $0(\mathrm{R})$ & $0(\mathrm{R})$ & $11(\mathrm{~S})$ & $0(\mathrm{R})$ & $21(\mathrm{~S})$ & Leuconostoc mesenteroides \\
\hline
\end{tabular}


Table 4. Cont.

\begin{tabular}{|c|c|c|c|c|c|c|c|c|c|c|c|c|c|}
\hline Car. & T80 & OLE & Lim. & C. HMW1 & C. HMW2 & C. MMW & C. LMW & A.A. & EEP 1 & EEP 2 & ETOH & Citrox ${ }^{\circledR}$ & Identified Isolate \\
\hline $0(\mathrm{R})$ & $0(\mathrm{R})$ & $0(\mathrm{R})$ & $0(\mathrm{R})$ & $0(\mathrm{R})$ & $0(\mathrm{R})$ & $0(\mathrm{R})$ & $0(\mathrm{R})$ & $12(\mathrm{~S})$ & $18(\mathrm{~S})$ & $12(\mathrm{~S})$ & $0(\mathrm{R})$ & $40(\mathrm{~S})$ & Leuconostoc miyukkimchii \\
\hline 12 (S) & $10(\mathrm{~S})$ & $7(\mathrm{R})$ & $10(\mathrm{~S})$ & $8(\mathrm{R})$ & $8(\mathrm{R})$ & $8(\mathrm{R})$ & $8(\mathrm{R})$ & $0(\mathrm{R})$ & $7(\mathrm{R})$ & $8(\mathrm{R})$ & $0(\mathrm{R})$ & $26(S)$ & Leuconostoc miyukkimchii \\
\hline $0(\mathrm{R})$ & $0(\mathrm{R})$ & $0(\mathrm{R})$ & $0(\mathrm{R})$ & $0(\mathrm{R})$ & $0(\mathrm{R})$ & $0(\mathrm{R})$ & $0(\mathrm{R})$ & $10(S)$ & $12(\mathrm{~S})$ & $12(\mathrm{~S})$ & $0(\mathrm{R})$ & $30(\mathrm{~S})$ & Leuconostoc miyukkimchii \\
\hline $0(\mathrm{R})$ & $0(\mathrm{R})$ & $0(\mathrm{R})$ & $0(\mathrm{R})$ & $0(\mathrm{R})$ & $0(\mathrm{R})$ & $0(\mathrm{R})$ & $0(\mathrm{R})$ & $8(\mathrm{R})$ & $12(\mathrm{~S})$ & $15(\mathrm{~S})$ & $0(\mathrm{R})$ & $28(S)$ & Leuconostoc miyukkimchii \\
\hline $0(\mathrm{R})$ & $0(\mathrm{R})$ & $0(\mathrm{R})$ & $0(\mathrm{R})$ & $0(\mathrm{R})$ & $0(\mathrm{R})$ & $0(\mathrm{R})$ & $0(\mathrm{R})$ & $10(\mathrm{~S})$ & $13(\mathrm{~S})$ & $10(\mathrm{~S})$ & $0(\mathrm{R})$ & $30(\mathrm{~S})$ & Leuconostoc miyukkimchii \\
\hline $0(\mathrm{R})$ & $0(\mathrm{R})$ & $0(\mathrm{R})$ & $0(\mathrm{R})$ & $11(\mathrm{~S})$ & $11(\mathrm{~S})$ & $10(\mathrm{~S})$ & $11(\mathrm{~S})$ & $11(\mathrm{~S})$ & $8(\mathrm{R})$ & $8(\mathrm{R})$ & 7 (R) & $20(\mathrm{~S})$ & Leuconostoc miyukkimchii \\
\hline $0(\mathrm{R})$ & $0(\mathrm{R})$ & $0(\mathrm{R})$ & $0(\mathrm{R})$ & $0(\mathrm{R})$ & $0(\mathrm{R})$ & $0(\mathrm{R})$ & $0(\mathrm{R})$ & $0(\mathrm{R})$ & $10(\mathrm{~S})$ & $12(\mathrm{~S})$ & $0(\mathrm{R})$ & $19(\mathrm{~S})$ & Leuconostoc miyukkimchii \\
\hline $0(\mathrm{R})$ & $0(\mathrm{R})$ & $0(\mathrm{R})$ & $0(\mathrm{R})$ & $0(\mathrm{R})$ & $0(\mathrm{R})$ & $0(\mathrm{R})$ & $0(\mathrm{R})$ & $10(S)$ & $14(\mathrm{~S})$ & $12(\mathrm{~S})$ & $0(\mathrm{R})$ & $34(\mathrm{~S})$ & Leuconostoc miyukkimchii \\
\hline $0(\mathrm{R})$ & $0(\mathrm{R})$ & $0(\mathrm{R})$ & $0(\mathrm{R})$ & $0(\mathrm{R})$ & $0(\mathrm{R})$ & $0(\mathrm{R})$ & $0(\mathrm{R})$ & $13(\mathrm{~S})$ & $16(\mathrm{~S})$ & $14(\mathrm{~S})$ & $0(\mathrm{R})$ & $38(\mathrm{~S})$ & Leuconostoc miyukkimchii \\
\hline $0(\mathrm{R})$ & $0(\mathrm{R})$ & $0(\mathrm{R})$ & $0(\mathrm{R})$ & $15(\mathrm{~S})$ & 15 (S) & $13(\mathrm{~S})$ & $15(\mathrm{~S})$ & $15(\mathrm{~S})$ & $8(\mathrm{R})$ & $8(\mathrm{R})$ & $0(\mathrm{R})$ & $25(\mathrm{~S})$ & Leuconostoc miyukkimchii \\
\hline $0(\mathrm{R})$ & $0(\mathrm{R})$ & $0(\mathrm{R})$ & $0(\mathrm{R})$ & $0(\mathrm{R})$ & $0(\mathrm{R})$ & $0(\mathrm{R})$ & $0(\mathrm{R})$ & $0(\mathrm{R})$ & $0(\mathrm{R})$ & $10(\mathrm{~S})$ & $0(\mathrm{R})$ & $28(\mathrm{~S})$ & Weissella fabalis \\
\hline $0(\mathrm{R})$ & $0(\mathrm{R})$ & $0(\mathrm{R})$ & $0(\mathrm{R})$ & $0(\mathrm{R})$ & $0(\mathrm{R})$ & $0(\mathrm{R})$ & $0(\mathrm{R})$ & $0(\mathrm{R})$ & $0(\mathrm{R})$ & $10(\mathrm{~S})$ & $0(\mathrm{R})$ & 26 (S) & Weissella beninensis \\
\hline $0(\mathrm{R})$ & $0(\mathrm{R})$ & $0(\mathrm{R})$ & $0(\mathrm{R})$ & $0(\mathrm{R})$ & $0(\mathrm{R})$ & $0(\mathrm{R})$ & $0(\mathrm{R})$ & $0(\mathrm{R})$ & $0(\mathrm{R})$ & $10(\mathrm{~S})$ & $0(\mathrm{R})$ & $24(\mathrm{~S})$ & Weissella fabalis \\
\hline $0(\mathrm{R})$ & $0(\mathrm{R})$ & $0(\mathrm{R})$ & $0(\mathrm{R})$ & $0(\mathrm{R})$ & $0(\mathrm{R})$ & $0(\mathrm{R})$ & $0(\mathrm{R})$ & $0(\mathrm{R})$ & $0(\mathrm{R})$ & $10(\mathrm{~S})$ & $0(\mathrm{R})$ & $20(S)$ & Weissella fabalis \\
\hline
\end{tabular}

Legend: Car. (carvacrol), T80 (polysorbate), Lim. (limonene), C. HMW (chitosan high molecular weight), MMW (medium molecular weight), LMW (low molecular weight), A.A. (acetic acid), EEP (ethanolic extract of propolis), ETOH (ethanol), R (resistant), S (sensitive). 
Sensitivity was assumed when an inhibition halo equal or superior to $10 \mathrm{~mm}$ in diameter was observed (Table 4 ). The percent sensitivity was calculated by dividing the number of inhibited isolates by the number of isolates from each type (Pseudomonas, Enterobacterales, or LAB) or by the total number of isolates. These results are presented in Table 5.

Table 5. Screening of antimicrobial activity of 10 natural compounds and respective controls.

\begin{tabular}{|c|c|c|c|c|}
\hline Compounds & $\%$ Sensitivity Pseudomonas & $\%$ Sensitivity Enterobacterales & $\%$ Sensitivity LAB & $\%$ Sensitivity Total \\
\hline $0.05 \%(v / v)$ Carvacrol & 0.0 & 0.0 & 3.8 & 1.0 \\
\hline Polysorbate 80 & 0.0 & 10.0 & 3.8 & 6.0 \\
\hline $1.5 \%(w / v)$ Olive leaf extract & 0.0 & 0.0 & 0.0 & 0.0 \\
\hline $0.05 \%(v / v)$ Limonene & 0.0 & 10.0 & 3.8 & 6.0 \\
\hline $3 \%(v / v)$ Chitosan High Weight 1 & 100.0 & 86.0 & 11.5 & 70.0 \\
\hline 3\% $(v / v)$ Chitosan High Weight 2 & 100.0 & 86.0 & 11.5 & 70.0 \\
\hline 3\% (v/v) Chitosan Medium Weight & 100.0 & 88.0 & 11.5 & 71.0 \\
\hline $3 \%(v / v)$ Chitosan Low Weight & 100.0 & 86.0 & 11.5 & 70.0 \\
\hline $3 \%(v / v)$ Acetic Acid & 100.0 & 88.0 & 46.2 & 80.0 \\
\hline $10 \%(w / v)$ EPE 1 & 0.0 & 0.0 & 46.2 & 12.0 \\
\hline $10 \%(w / v)$ EPE 2 & 0.0 & 0.0 & 73.1 & 19.0 \\
\hline $70 \%(v / v)$ Ethanol & 0.0 & 2.0 & 0.0 & 1.0 \\
\hline $50 \%(v / v)$ Citrox $^{\circledR}$ & 100.0 & 100.0 & 100.0 & 100.0 \\
\hline
\end{tabular}

Legend: EPE-ethanolic propolis extract.

Of all the products investigated, only Citrox ${ }^{\circledR}$ inhibited all of the selected isolates (Tables 4 and 5). For this reason, only minimum inhibitory concentrations of the Citrox ${ }^{\circledR}$ solution were further determined. Concentrations ranging from $0.03 \%$ to $50 \%(v / v)$ Citrox ${ }^{\circledR}$ solution were prepared with sterile deionized water, and minimum inhibitory concentrations were determined. The results obtained are shown in Table 6.

Table 6. Inhibitory effect of Citrox ${ }^{\circledR}$ solutions at different concentrations.

\begin{tabular}{cc}
\hline$\%(v / v)$ Citrox $^{\circledR}$ & \% Sensitivity \\
\hline 50.00 & 100 \\
25.00 & 100 \\
12.50 & 100 \\
6.75 & 100 \\
3.38 & 100 \\
1.69 & 100 \\
0.84 & 53 \\
0.42 & 17 \\
0.21 & 13 \\
0.11 & 8 \\
0.06 & 3 \\
0.03 & 3 \\
\hline
\end{tabular}

\section{Discussion}

\subsection{Microbiological Characterisation of Squid and Shrimp Skewers}

No L. monocytogenes or E. coli were detected in the analyzed products. Despite not being common, the presence of L. monocytogenes [34] and E. coli [10] in seafood may occur [35], and the contamination by these pathogens is usually associated with unsafe handling of food during processing [11]. The absence of E. coli and L. monocytogenes suggests that safety procedures may have been applied during production.

Enumeration performed immediately after reception of the product showed a high microbial load for all parameters tested. Pseudomonas spp. seemed to be the most abundant type of bacteria with over $6 \log \mathrm{CFU} / \mathrm{g}$ at the moment of reception, followed by members of the Enterobacterales order, with over $4 \log \mathrm{CFU} / \mathrm{g}$, and LAB, with just under $4 \log \mathrm{CFU} / \mathrm{g}$. Pseudomonas spp. are among the most noteworthy spoiling organisms in seafood [36].

The storage of the product under refrigerated conditions resulted in a considerable growth of bacterial communities during the five days of testing, with an increase of 3 to 
$4 \log$ CFU/g observed for all the microbiological parameters. This increase was mainly observed on the second day of storage. This contrasts with what was observed by Don et al. [37] and Farajzadeh et al. [38], where microbial levels of shrimp only surpassed $8 \log$ CFU/g after 12-14 and 8-10 days, respectively. This can be explained by these studies focusing exclusively on shrimp and because some irregularity in bacterial counts is expected depending on the capture zone and processing facility. Moreover, the skewers analyzed in the present study are subject to considerably more human manipulation than the food matrices studied by Don et al. [37] and Farajzadeh et al. were [38].

\subsection{Identification of Selected Isolates by $16 S$ rRNA Sequencing}

Of the 100 randomly selected isolates, 50 were identified as members of the Enterobacterales order (according to Adeolu et al. [39]), being these distributed by the Enterobacteriaceae (Klebsiella genus), Hafniaceae (Hafnia and Obesumbacterium genus), Yersiniaceae (Serratia and Rahnella genus), Morganellaceae (Morganella genus), and Erwiniaceae (Pantoea genus) families [39]. Bacteria of the Serratia genus (Serratia liquefaciens, Serratia nematodiphila, and Serratia fonticola), commonly found in the environment, such as in soil, water, and intestinal tract of digestive tracts of various animals, represented over $20 \%$ of all isolates identified in the studied product. The facultative anaerobic bacteria S. liquefaciens, after Serratia marcescens, is the most common Serratia species involved in human infections, being previously connected with non-foodborne infections. [40]. Fourteen isolates were identified as Rahnella spp., another genus of the Yersiniaceae family, that can be found in soil, water, and intestinal tracts of herbivores [41]. Rahnella aquatilis is a fish pathogen that induces hemorrhagic septicemias in vulnerable hosts. In humans, R. aquatilis is an opportunistic pathogen since it can cause various infections, from wound infection to sepsis [41]. Two isolates were also identified as Klebsiella oxytoca, which is recognized as a pathogen of interest, due to its opportunistic characteristics and resistance to antibiotics [42]. This bacterium is usually present in water and soil and can be found in the intestine tracts of several animals, including humans [42]. Its presence in ready-to-eat food products has been previously reported by Nyenje et al. [43], alerting to unsafe practices that can pose serious threats to consumers. Hafnia alvei and Morganella morganii were also detected in the samples analyzed ( 6 and 3 isolates, respectively), and, as reported by Montet and Ray [6], these organisms are responsible for the production of biogenic amines in food products.

None of the 24 isolates of Pseudomonas spp. found are known to be human pathogens, being all the species widespread in various environments [44]. However, Pseudomonas spp., and more specifically Pseudomonas fragi (two isolates identified as P. fragi), comprise one of the most relevant genera responsible for the spoilage of shrimp in cold storage conditions [7]. The presence of this microorganism and its production of enzymes leads to the decomposition of the product and the surge of unpleasant sensorial changes [36]. While none of the isolates of Pseudomonas were identified as Pseudomonas aeruginosa, the most significant opportunistic pathogen of this genus [36], the presence of several species of this genus indicates the existence of ideal growing conditions for the proliferation of such a spoiling-inducing genus in this product. Any shelf-life-extending technique or compound to be applied to this shrimp and squid skewer should strive for the inhibition or elimination of Pseudomonas growth and microbial load.

Regarding LAB, Lactococcus spp. and Leuconostoc spp. were found in high numbers. Leuconostoc spp. are mesophilic and psychrotolerant bacteria of the Lactobacillaceae family, and some species such as Leuconostoc gelidum are commonly found in meat and fish products [45]. Production of lactic and acetic acids by Leuconostoc spp. through the fermentation of carbohydrates is a cause of food spoilage. Formation of slime, discoloration, and alteration of smells and flavors are also products of Leuconostoc activity $[46,47]$. While negative effects on food products can ensue from the proliferation of these bacteria, some Leuconostoc mesenteroides and Leuconostoc lactis strains have been recorded as potentially probiotic and bacteriocins producers, having antibacterial activity against the foodborne pathogen L. monocytogenes [48-50]. Lactococcus piscium was previously identified as a fish 
pathogen in diseased rainbow trout [51]. However, the relation between the presence of this bacteria and the disease has never been established [52], unlike its relation to food spoilage. This spoilage is, however, dependent on the strain and the type of foodstuff; Lac. piscium is known to cause the spoilage of meat but seems to have no impact on the degradation of seafood [52]. Lactococcus gerviae has been shown to cause "septicemias, ophthalmia, and hemorrhages" in marine farmed fish. In humans, Lac. gerviae has been the cause of some infections; its proliferation in the human body can possibly lead to infective endocarditis $[53,54]$.

\subsection{Antimicrobial Activity Screening of Each Natural Compound}

Measurement of the diameter of the inhibition halos was performed, and, according to García-Díez et al. [55], only halos superior to $10 \mathrm{~mm}$ of diameter were defined as indicative of susceptibility of a given isolate to a specific compound (Table 4). Due to their antimicrobial activity and functional properties, essential oils, such as limonene, thymol, oleuropein, and carvacrol, have been the focus of several studies [15,17-20]. Solutions of $0.05 \%(v / v)$ carvacrol and limonene showed antimicrobial activity against $1.0 \%$ and $6.0 \%$ of the isolates, respectively (Tables 4 and 5). Isolates that were inhibited by limonene were also inhibited by the emulsifier polysorbate 80, whereby this inhibition was not considered. Contrary to what was found in this study, Bunyan et al. [56] described carvacrol as an efficient antimicrobial agent against Serratia spp. with minimum inhibitory concentrations below $0.04 \%(v / v)$. Thielmann and Muranyi [57] reported minimum inhibitory concentrations of limonene for Klebsiella spp., Leuconostoc spp., and Pseudomonas spp. of 1.1\%, 2.0\%, and $0.8 \%(v / v)$, respectively. This indicates that in order to inhibit bacterial growth, much higher concentrations of limonene would have to be used. Curiously, the presence of these compounds in the disk appeared to improve the bacterial growth of some isolates. This occurred rarely, nonetheless, increased bacterial growth was observed 3-4 $\mathrm{mm}$ from the periphery of the disk. This result suggests that at some unknown concentration, polysorbate might induce bacterial proliferation. Similar findings were observed in the study by Nielsen et al. [58], where polysorbate $80(0.1 \% \mathrm{v} / \mathrm{v})$ added to the growth medium increased the growth rate of S. aureus while reducing the growth of L. monocytogenes. Nielsen et al. [58] also reported that the presence of polysorbate 80 reduced antimicrobial activity of both rifampicin and isoeugenol, indicating that polysorbate could be inhibiting the antibacterial activity of limonene, carvacrol, and olive leaf extract used in this present study.

Due to its nontoxic, biocompatible, safe-to-use, biodegradable, and antibacterial characteristics, chitosan has been suggested as a strong candidate for application in food products [59-61]. Chitosan is, however, only an efficient antimicrobial agent in acidic conditions, due to its solubility in low pH media [59]. In this study, all 3\% (w/v) chitosan acidic solutions appeared to inhibit over $70 \%$ of the isolates (Tables 4 and 5). The totality of Pseudomonas spp. and over $80 \%$ of isolates identified as belonging to the Enterobacterales order showed susceptibility to all four chitosan solutions tested. This agrees with the study of Cao et al. [62], where chitosan was also analyzed in the context of the shelf-life extension of molluscs. In that study, chitosan at $0.5 \%(w / v)$ was an effective antimicrobial against most isolates tested, including Pseudomonas spp. and Enterobacterales. While, in the present study, chitosan appeared to inhibit Pseudomonas spp. and Enterobacterales growth, the same isolates were also inhibited by the 3\% (v/v) acetic acid used as control (Tables 4 and 5 ). Because of this, it is not clear if the inhibition of bacterial growth was exclusively the result of the acetic acid in the solution. Therefore, the analysis of water-soluble chitosan might provide clearer information regarding the efficiency of chitosan, since the presence of acetic acid seems to overshadow the antibacterial activity of chitosan. Cao et al. [62] also observed inhibition of LAB isolates by a chitosan $0.1 \%(w / v)$ solution, with halos superior to $15 \mathrm{~mm}$. In the present study, the chitosan acidic solution appeared to have a suboptimal antibacterial activity against LAB. It is speculated that this result is a consequence of a high resistance of $\mathrm{LAB}$ to acidic environments. 
Propolis is a resinous mixture of beeswax and other resins collected by honeybees, usually of the Apis melifera species. It provides structural and protective action to the honeycomb [63]. While a strong antibacterial activity is commonly found as a characteristic of this compound [17], its antimicrobial activity is known to vary accordingly to the environmental characteristics from which it originates [63]. Ethanolic propolis extracts at $10 \%(w / v)$ inhibited the bacterial growth of some isolates, but this inhibition varied depending on the propolis used, being EPE2 slightly more efficient than EPE1. While inhibiting bacterial growth of some isolates, all of the susceptible isolates were LAB, which indicates that these solutions, while being efficient in controlling LAB proliferation, lack antibacterial activity against Pseudomonas and Enterobacterales. The results obtained are supported by the lack of bacterial inhibition by the ethanolic solution used as control, showing that antibacterial activity is a consequence of the propolis properties. Przybyłek and Karpiński [63] presented a compendium of studies of antibacterial activity of ethanolic propolis extracts against several bacteria with minimum inhibitory concentrations ranging from $5 \mu \mathrm{g} / \mathrm{mL}$, against Streptococcus sobrinius, to over $2900 \mu \mathrm{g} / \mathrm{mL}$, against Salmonella spp. While there is a considerable range of concentrations, the solution used in this study is considerably superior to those observed as efficient in the study of Przybyłek and Karpiński [63]. This might be the result of the known variability of propolis composition relative to the environmental conditions of where it is produced.

Citrox ${ }^{\circledR}$ ProGarda solution managed to inhibit the growth of all isolates. This result presents Citrox ${ }^{\circledR}$ as the product of interest, due to its broad-range activity capable of controlling bacterial proliferation in all isolates tested. Taking into account its composition based on a citrus extract, with phenolic compounds and organic acids as its active ingredients, microbial inhibition is due to both $\mathrm{pH}$ regulation and bioflavonoid activity. Tsiraki et al. [64-66] observed reductions in colony counts of Bacillus cereus [65], Salmonella enterica [65], and Escherichia coli O157:H7 [66] in Tzatziki, a traditional Greek yogurt-based salad, after the addition of Citrox ${ }^{\circledR}$ ProGarda. Shelf-life extension of this traditional dish due to the impact of Citrox ${ }^{\circledR}$ was also achieved [64]. Yehia et al. [67] also observed the total reduction of volatile basic nitrogen and methicillin-resistant $S$. aureus values in chicken meat after application of a Citrox ${ }^{\circledR}$ solution; however, the solution used in this study had a different formulation. Since the purpose of this study was to collect information on natural compounds for their future use in the shelf-life extension of squid and shrimp skewers, of the above-mentioned solutions, only the application of a Citrox ${ }^{\circledR}$ solution presented the recommended activity for the proposed objective.

\subsection{Minimum Inhibitory and Bactericidal Concentrations of Citrox ${ }^{\circledR}$}

Regarding the minimum inhibitory concentration of Citrox ${ }^{\circledR}$, when diluted to a final concentration of $1.69 \%(v / v)$ or higher, Citrox ${ }^{\circledR}$ inhibited all the isolates tested. Citrox ${ }^{\circledR}$ antibacterial activity decreased with increasing dilutions, inhibiting bacterial growth of only three isolates at the minimum concentration of $0.03 \%$. Since the maximum recommended concentration for the spraying of foods with this product is $3 \%(v / v)$, the minimal inhibitory concentration observed was considered sufficient to achieve the proposed objective. To our knowledge, no studies about the MICs of Citrox ${ }^{\circledR}$ ProGarda against the same species identified in this study are available in the literature. However, other formulations of Citrox ${ }^{\circledR}$ are used for the disinfection of different surfaces, such as Citrox ${ }^{\circledR}$ BC30 and Citrox ${ }^{\circledR}$ MDC30 [68]. Different combinations of bioflavonoids with malic and citric acids cause the variation of its antibacterial activities; $2 \%(v / v)$ Citrox ${ }^{\circledR}$ BC30 showed activity against Clostridium difficile while $8 \%(v / v)$ Citrox ${ }^{\circledR}$ MDC30 was unable to inhibit the same pathogen [68].

All the suspensions where no growth was observed were plated on Müeller-Hinton Agar, and bacterial growth was observed in 97 out of the 100 isolates tested. This result indicates that Citrox ${ }^{\circledR}$ at $1.69 \%$ mainly acted as a bacteriostatic compound, inhibiting bacterial growth but not destroying all bacterial cells. Considering that the shelf-life of the product is considerably short ( 3 to 4 days), and the purpose of any treatment is to extend it, 
the bacteriostatic properties of Citrox ${ }^{\circledR}$ were deemed sufficient to the purpose intended, since the inhibition of bacterial growth and consequential spoilage for an additional 1 or 2 days would already considerably augment the freshness period and shelf-life of such perishable product.

\section{Conclusions}

In the present study, microbial characterization of squid and shrimp skewers was performed. Considerably high total viable counts were observed in the product. Counts on Pseudomonas-selective agar revealed that bacteria of this genus were the most numerous in the product. The high spoilage activity of Pseudomonas and its levels in this product confirm its fast perishability. Listeria monocytogenes and E. coli were not detected, which supports the idea that the processing of the product follows proper safety guidelines. One hundred selected bacteria were identified as Enterobacterales, Pseudomonas, or LAB, which shows a complete dominance of these bacterial groups in the microbiome of the product. Antimicrobial activity of limonene, carvacrol, olive leaf extract, propolis, Citrox ${ }^{\circledR}$, and chitosan was tested against the isolated microorganisms. Solutions of limonene, carvacrol, and olive leaf extract lacked noteworthy antibacterial activity against the isolates tested. While all three chitosan solutions appeared to inhibit growth of a significant proportion of the isolates $(>70 \%)$, the same activity was observed in the control solution. Ethanolic propolis extracts showed some efficiency in inhibiting LAB growth, but lacked antibacterial activity against the remaining isolates. Only Citrox ${ }^{\circledR}$ inhibited the growth of all isolates, displaying bacteriostatic and bactericidal activity to $97 \%$ and $3 \%$ of the isolates, respectively, at concentrations higher or equal to $1.69 \%(v / v)$. While the need for further tests to prove this theory remains, the application of Citrox ${ }^{\circledR}$ to seafood products presents itself as a potential shelf-life-extending strategy.

Author Contributions: Conceptualization, L.P.d.R., J.B.B., D.F.C., A.M.S. and P.T.; formal analysis, L.P.d.R. and J.B.B.; investigation, L.P.d.R. and J.B.B.; resources, P.T., A.M.S. and D.F.C.; writingoriginal draft preparation, L.P.d.R. and J.B.B.; writing-review and editing, L.P.d.R., J.B.B., P.T., D.F.C., A.M.S. and A.M.G.; supervision, J.B.B. and P.T.; project administration, A.M.G.; funding acquisition, P.T. All authors have read and agreed to the published version of the manuscript.

Funding: This research was funded by Fundo Europeu de Desenvolvimento Regional (FEDER) by Programa Operacional Competitividade e Internacionalização (POCI) through the research project VALORMAR: Valorização Integral dos Recursos Marinhos: Potencial, Inovação Tecnológica e Novas Aplicações (POCI-01-0247-FEDER-024517).

Data Availability Statement: The datasets generated for this study are available on request to the corresponding author.

Acknowledgments: The authors would also like to thank the scientific collaboration under the Fundação para a Ciência e a Tecnologia (FCT) project UIDP/00329/2020. Financial support for author J. Barbosa was provided by a postdoctoral fellowship SFRH/BPD/113303/2015 (FCT).

Conflicts of Interest: This work was developed in the scope of the project ValorMar (https:// valormar.pt/en/project/, accessed on 15 February 2022) and SONAE is a partner of this project (https://valormar.pt/en/partners/, accessed on 15 February 2022). And the authors declare no conflict of interest.

\section{References}

1. Wu, L.; Pu, H.; Sun, D.W. Novel techniques for evaluating freshness quality attributes of fish: A review of recent developments. Trend Food Sci. Technol. 2019, 83, 259-273. [CrossRef]

2. Møretrø, T.; Moen, B.; Heir, E.; Hansen, A.; Langsrud, S. Contamination of salmon fillets and processing plants with spoilage bacteria. Int. J. Food Microbiol. 2016, 237, 98-108. [CrossRef] [PubMed]

3. Fidalgo, L.G.; Lemos, Á.T.; Delgadillo, I.; Saraiva, J.A. Microbial and physicochemical evolution during hyperbaric storage at room temperature of fresh Atlantic salmon (Salmo salar). Innov. Food Sci. Emerg. Technol. 2018, 45, 264-272. [CrossRef] 
4. Giarratana, F.; Muscolino, D.; Beninati, C.; Ziino, G.; Giuffrida, A.; Panebianco, A. Activity of R(+) limonene on the maximum growth rate of fish spoilage organisms and related effects on shelf-life prolongation of fresh gilthead sea bream fillets. Int. J. Food Microbiol. 2016, 237, 109-113. [CrossRef]

5. Boziaris, I.S.; Parlapani, F.F. Specific Spoilage Organisms (SSOs) in Fish. In The Microbiological Quality of Food: Foodborne Spoilers, 1st ed.; Bevilacqua, A., Corbo, M.R., Sinigaglia, M., Eds.; Woodhead Publishing: Sawston, UK, 2017; pp. 61-98. [CrossRef]

6. Leroi, F.; Joffraud, J. Microbial Degradation of Seafood. In Aquaculture Microbiology and Biotechnology, 1st ed.; Montet, D., Ray, R.C., Eds.; Science Publishers: Enfield, NH, USA, 2009; Volume 1, pp. 47-57.

7. Bolívar, A.; Costa, J.; Posada, G.; Pérez-Rodríguez, F.; Bascón Villeas, I.; Valero, A. Characterization of Foodborne Pathogens and Spoilage Bacteria in Mediterranean Fish Species and Seafood Products. In Food Borne Pathogens and Antibiotic Resistance, 1st ed.; Singh, O.V., Ed.; Wiley-Blackwell: Hoboken, NJ, USA, 2017; Volume 1, pp. 21-34.

8. Iwamoto, M.; Ayers, T.; Mahon, B.E.; Swerdlow, D.L. Epidemiology of Seafood-Associated Infections in the United States. Clin. Microbiol. Rev. 2010, 23, 399-411. [CrossRef]

9. Jami, M.; Ghanbari, M.; Zunabovic, M.; Domig, K.J.; Kneifel, W. Listeria monocytogenes in aquatic food products-A review. Comp . Rev. Food Sci. Food Safety 2014, 13, 798-813. [CrossRef]

10. Costa, R.A. Escherichia coli in seafood: A brief overview. Adv. Biosci. Biotechnol. 2013, 4, 450-454. [CrossRef]

11. Vongkamjan, K.; Benjakul, S.; Kim, V.H.T.; Vuddhakul, V. Longitudinal monitoring of Listeria monocytogenes and Listeria phages in seafood processing environments in Thailand. Food Microbiol. 2017, 66, 11-19. [CrossRef]

12. Getu, A.; Misganaw, K. Post-harvesting and Major Related Problems of Fish Production. Fisher. Aquacult. J. 2015, 6, 4. [CrossRef]

13. Al-Maqtari, Q.A.; Rehman, A.; Mahdi, A.A. Application of essential oils as preservatives in food systems: Challenges and future prospectives-A review. Phytochem. Rev. 2021, Volume, Page. [CrossRef]

14. Kulawik, P.; Tiwari, B.K. Recent advancements in the application of non-thermal plasma technology for the seafood industry. Crit. Rev. Food Sci. Nutrition. 2019, 59, 3199-3210. [CrossRef] [PubMed]

15. Han, Y.; Sun, Z.; Chen, W. Antimicrobial Susceptibility and Antibacterial Mechanism of Limonene against Listeria monocytogenes. Molecules 2020, 25, 33. [CrossRef] [PubMed]

16. Rezaei, F.; Shahbazi, Y. Shelf-life extension and quality attributes of sauced silver carp fillet: A comparison among direct addition, edible coating and biodegradable film. LWT Food Sci. Technol. 2018, 87, 122-133. [CrossRef]

17. Casquete, R.; Castro, S.M.; Jácome, S.; Teixeira, P. Antimicrobial activity of ethanolic extract of propolis in "Alheira", a fermented meat sausage. Cogent. Food Agric. 2016, 2. [CrossRef]

18. Himour, S.; Yahia, A.; Belattar, H. Oleuropein and Antibacterial Activities of Olea europaea L. Leaf Extract. Eur. Sci. J. 2017, 13, 342. [CrossRef]

19. Marchese, A.; Orhan, I.E.; Daglia, M. Antibacterial and antifungal activities of thymol: A brief review of the literature. Food Chem. 2016, 210, 402-414. [CrossRef]

20. Ozogul, Y.; Kuley Boğa, E.; Akyol, I. Antimicrobial activity of thyme essential oil nanoemulsions on spoilage bacteria of fish and food-borne pathogens. Food Biosci. 2020, 36. [CrossRef]

21. Bertuzzi, M.A.; Slavutsky, A.M. Standard and New Processing Techniques Used in the Preparation of Films and Coatings at the Lab Level and Scale-Up. In Edible Films and Coatings: Fundamentals and Applications, 1st ed.; Garcia, M.P.M., Gómez-Guillén, M.C., López-Caballero, M.E., Barbosa-Cánovas, G.V., Eds.; CRC Press: Boca Raton, FL, USA, 2017; pp. 3-10.

22. Alagawany, M.; Farag, M.R.; Abdelnour, S.A.; Elnesr, S.S. A review on the beneficial effect of thymol on health and production of fish. Rev. Aquacult. 2021, 13, 632-641. [CrossRef]

23. Iso 4833-1:2013-Microbiology of the Food Chain-Horizontal Method For The Enumeration Of Microorganisms-Part 1: Colony Count At $30{ }^{\circ} \mathrm{C}$ By The Pour Plate Technique. 2021. Available online: https:/ /www.iso.org/standard/53728.html (accessed on 5 December 2021).

24. ISO 15214:1998-Microbiology of Food and Animal Feeding Stuffs_-Horizontal Method for the Enumeration Of Mesophilic Lactic Acid Bacteria-Colony-Count Technique at 30 Degrees C. 2021. Available online: https://www.iso.org/standard/26853.html (accessed on 5 December 2021).

25. ISO 13720:2010-Meat and Meat Products-Enumeration of Presumptive Pseudomonas spp. 2021. Available online: https: //www.iso.org/standard/45099.html (accessed on 5 December 2021).

26. ISO 21528-2:2017-Microbiology of the Food Chain-Horizontal Method for the Detection and Enumeration of EnterobacteriaceaePart 2: Colony-count technique. 2021. Available online: https://www.iso.org/standard/63504.html (accessed on 5 December 2021).

27. ISO 11290-2:2017-Microbiology of the Food Chain-Horizontal Method for the Detection and Enumeration of Listeria monocytogenes and of Listeria spp.-Part 2: Enumeration Method. 2021. Available online: https://www.iso.org/standard/60314.html (accessed on 5 December 2021).

28. ISO 16649-1:2018-Microbiology of the Food Chain-Horizontal Method for the Enumeration of beta-glucuronidase-positive Escherichia coli-Part 1: Colony-cOunt Technique at $44^{\circ} \mathrm{C}$ Using Membranes and 5-bromo-4-chloro-3-indolyl beta-D-glucuronide. 2021. Available online: https://www.iso.org/standard/64951.html (accessed on 5 December 2021).

29. Vaz-Moreira, I.; Faria, C.; Lopes, A.R.; Svensson, L.; Falsen, E.; Moore, E.R.; Ferreira, A.C.; Nunes, O.C.; Manaia, C.M. Sphingobium vermicomposti sp. nov., isolated from vermicompost. Int. J. Syst. Evol. Microbiol. 2009, 59, 3145-3149. [CrossRef] 
30. Altschul, S.F.; Madden, T.L.; Schäffer, A.A. Gapped BLAST and PSI-BLAST: A New Generation of Protein Database Search Programs. In Nucleic Acids Research; Oxford University Press: Oxford, UK, 1997; Volume 25, pp. 3389-3402. [CrossRef]

31. Casquete, R.; Castro, S.M.; Teixeira, P. Evaluation of the Combined Effect of Chitosan and Lactic Acid Bacteria in Alheira (Fermented Meat Sausage) Paste. J. Food Proc. Preserv. 2017, 41, e12866. [CrossRef]

32. Bauer, A.W.; Kirby, W.M.M.; Sherris, J.C.; Turck, M. Antibiotic Susceptibility Testing by a Standardized Single Disk Method. Am. J. Clin. Pathol. 1966, 45, 493-496. [CrossRef] [PubMed]

33. Aumeeruddy-Elalfi, Z.; Gurib-Fakim, A.; Mahomoodally, M.F. Chemical composition, antimicrobial and antibiotic potentiating activity of essential oils from 10 tropical medicinal plants from Mauritius. J. Herb. Med. 2016, 6, 88-95. [CrossRef]

34. Das, S.; Lalitha, K.V.; Thampuran, N.; Surendran, P.K. Isolation and characterization of Listeria monocytogenes from tropical seafood of Kerala, India. Ann. Microbiol. 2012, 63, 1093-1098. [CrossRef]

35. Dumen, E.; Ekici, G.; Ergin, S.; Bayrakal, G.M. Presence of Foodborne Pathogens in Seafood and Risk Ranking for Pathogens. Foodborne Pathog. Dis. 2020, 17, 541-546. [CrossRef] [PubMed]

36. Raposo, A.; Pérez, E.; de Faria, C.T.; Ferrús, M.A.; Carrascosa, C. Food Spoilage by Pseudomonas spp.-An Overview. In Foodborne Pathogens and Antibiotic Resistance; Singh, O.V., Ed.; Wiley Blackwell: Hoboken, NJ, USA, 2017; pp. 41-71. [CrossRef]

37. Don, S.; Xavier, K.A.M.; Devi, S.T.; Nayak, B.B.; Kannuchamy, N. Identification of potential spoilage bacteria in farmed shrimp (Litopenaeus vannamei): Application of Relative Rate of Spoilage models in shelf life-prediction. LWT 2018, 97, 295-301. [CrossRef]

38. Farajzadeh, F.; Motamedzadegan, A.; Shahidi, S.A.; Hamzeh, S. The effect of chitosan-gelatin coating on the quality of shrimp (Litopenaeus vannamei) under refrigerated condition. Food Control 2016, 67, 163-170. [CrossRef]

39. Adeolu, M.; Alnajar, S.; Naushad, S.; Gupta, R.S. Genome-based phylogeny and taxonomy of the "Enterobacteriales": Proposal for Enterobacterales ord. nov. divided into the families Enterobacteriaceae, Erwiniaceae fam. nov., Pectobacteriaceae fam. nov., Yersiniaceae fam. nov., Hafniaceae fam. nov., Morganellaceae fam. nov., and Budviciaceae fam. nov. Int. J. Syst. Evol. Microbiol. 2016, 66, 5575-5599. [CrossRef]

40. Mahlen, S.D. Serratia infections: From military experiments to current practice. Clin. Microbiol. Rev. 2011, 24, 755-791. [CrossRef] [PubMed]

41. Liang, J.; Hu, X.; Aijun, L.; Sun, J. First report on the characterization of pathogenic Rahnella aquatilis KCL-5 from crucian carp: Revealed by genomic and proteomic analyses. J. Fish Dis. 2020, 43, 889-914. [CrossRef]

42. Singh, L.; Cariappa, M.P.; Kaur, M. Klebsiella oxytoca: An emerging pathogen? Med. J. Armed Forces India 2016, 72, 59-61. [CrossRef]

43. Nyenje, M.E.; Odjadjare, C.E.; Tanih, N.F.; Green, E.; Ndip, R.N. Foodborne pathogens recovered from ready-to-eat foods from roadside cafeterias and retail outlets in alice, eastern cape province, South Africa: Public health implications. Int. J. Environ. Res. Publ. Health 2012, 9, 2608-2619. [CrossRef] [PubMed]

44. Silby, M.W.; Winstanley, C.; Godfrey, S.A.C.; Levy, S.B.; Jackson, R.W. Pseudomonas genomes: Diverse and adaptable. FEMS Microbiol. Rev. 2011, 35, 652-680. [CrossRef] [PubMed]

45. Endo, A.; Maeno, S.; Liu, S.Q. Lactic Acid Bacteria: Leuconostoc spp. In Encyclopedia of Dairy Sciences, 3rd ed.; McSweeney, P., McNamara, J., Eds.; Academic Press: Boston, MA, USA, 2022; Volume 4, pp. 226-232. [CrossRef]

46. Wang, H.Y.; Wen, C.F.; Chiu, Y.H. Leuconostoc mesenteroides Growth in Food Products: Prediction and Sensitivity Analysis by Adaptive-Network-Based Fuzzy Inference Systems. PLoS ONE 2013, 8, e64995. [CrossRef] [PubMed]

47. Feiner, G. The microbiology of specific bacteria. In Meat Products Handbook, 1st ed.; Feiner, G., Ed.; Woodhead Publishing: Sawston, UK, 2006; pp. 595-615. [CrossRef]

48. Oliveira, M.; Barbosa, J.; Albano, H.; Teixeira, P. Bacteriocinogenic activity of Leuconostoc lactis RK18 isolated from fermented food. In Fermented Foods: Nutrition and Role in Health and Disease, 1st ed.; Kovalyov, O., Ed.; Nova Science Publishers: New York, NY, USA, 2020; pp. 159-181.

49. de Paula, A.T.; Jeronymo-Ceneviva, A.B.; Todorov, S.D.; Penna, A.L.B. The Two Faces of Leuconostoc mesenteroides in Food Systems. Food Rev. Int. 2015, 31, 147-171. [CrossRef]

50. Pinto, A.; Barbosa, J.; Albano, H.; Isidro, J.; Teixeira, P. Screening of bacteriocinogenic lactic acid bacteria and their characterization as potential probiotics. Microorganisms 2020, 8, 393. [CrossRef] [PubMed]

51. Williams, A.M.; Fryer, J.L.; Collins, M.D. Lactococcus piscium sp. nov. a new Lactococcus species from salmonid fish. FEMS Microbiol. Lett. 1990, 68, 109-113. [CrossRef]

52. Saraoui, T.; Leroi, F.; Björkroth, J.; Pilet, M.F. Lactococcus piscium: A psychrotrophic lactic acid bacterium with bioprotective or spoilage activity in food-A review. J. Appl. Microbiol. 2016, 121, 907-918. [CrossRef]

53. Malek, A.; de la Hoz, A.; Gomez-Villegas, S.I.; Nowbakht, C.; Arias, C.A. Lactococcus garvieae, an unusual pathogen in infective endocarditis: Case report and review of the literature. BMC Infect. Dis. 2019, 19, 301. [CrossRef]

54. Zheng, J.; Wittouck, S.; Salvetti, E. A taxonomic note on the genus Lactobacillus: Description of 23 novel genera, emended description of the genus Lactobacillus beijerinck 1901, and union of Lactobacillaceae and Leuconostocaceae. Int. J. Syst. Evol. Microbiol. 2020, 70, 2782-2858. [CrossRef]

55. García-Díez, J.; Alheiro, J.; Falco, V.; Fraqueza, M.J.; Patarata, L. Chemical characterization and antimicrobial properties of herbs and spices essential oils against pathogens and spoilage bacteria associated to dry-cured meat products. J. Essent. Oil Res. 2016, 29, 117-125. [CrossRef]

56. Bunyan, I.; Aumaima, T.; Abid, H.O. Antibacterial Activity of Carvacrol against Different Types of Bacteria. J. Nat. Sci. Res. 2014, $4,13-16$. 
57. Thielmann, J.; Muranyi, P. Review on the chemical composition of Litsea cubeba essential oils and the bioactivity of its major constituents citral and limonene. J. Essent. Oil Res. 2019, 31, 361-378. [CrossRef]

58. Nielsen, C.K.; Kjems, J.; Mygind, T.; Snabe, T.; Meyer, R.L. Effects of Tween 80 on Growth and Biofilm Formation in Laboratory Media. Front. Microbiol. 2016, 7, 1878. [CrossRef] [PubMed]

59. Sahariah, P.; Másson, M. Antimicrobial Chitosan and Chitosan Derivatives: A Review of the Structure-Activity Relationship. Biomacromolecules 2017, 18, 3846-3868. [CrossRef]

60. Haghighi, H.; Licciardello, F.; Fava, P.; Siesler, H.W.; Pulvirenti, A. Recent advances on chitosan-based films for sustainable food packaging applications. Food Pack. Shelf Life 2020, 26. [CrossRef]

61. Qu, B.; Luo, Y. Chitosan-based hydrogel beads: Preparations, modifications and applications in food and agriculture sectors-A review. Int. J. Biol. Macromol. 2020, 152, 437-448. [CrossRef]

62. Cao, R.; Xue, C.H.; Liu, Q. Changes in microbial flora of Pacific oysters (Crassostrea gigas) during refrigerated storage and its shelf-life extension by chitosan. Int. J. Food Microbiol. 2009, 131, 272-276. [CrossRef] [PubMed]

63. Przybyłek, I.; Karpiński, T.M. Antibacterial Properties of Propolis. Molecules 2019, 24, 2047. [CrossRef] [PubMed]

64. Tsiraki, M.I.; Savvaidis, I.N. Citrus extract or natamycin treatments on "Tzatziki"-A traditional Greek salad. Food Chemistry 2014, 142, 416-422. [CrossRef] [PubMed]

65. Tsiraki, M.I.; Savvaidis, I.N. The effects of citrus extract (Citrox@) on the naturally occurring microflora and inoculated pathogens, Bacillus cereus and Salmonella enterica, in a model food system and the traditional Greek yogurt-based salad Tzatziki. Food Microbiol. 2016, 53, 150-155. [CrossRef]

66. Tsiraki, M.I.; Yehia, H.M.; Elobeid, T.; Osaili, T.; Sakkas, H.; Savvaidis, I.N. Viability of and Escherichia coli O157:H7 and Listeria monocytogenes in a delicatessen appetizer (yogurt-based) salad as affected by citrus extract (Citrox@) and storage temperature. Food Microbiol. 2018, 69, 11-17. [CrossRef] [PubMed]

67. Yehia, H.M.; Elkhadragy, M.F.; Al-Masoud, A.H.; Al-Megrin, W.A. Citrox Improves the Quality and Shelf Life of Chicken Fillets Packed under Vacuum and Protects against Some Foodborne Pathogens. Animals 2019, 9, 1062. [CrossRef] [PubMed]

68. Hooper, S.J.; Lewis, M.A.O.; Wilson, M.J.; Williams, D.W. Antimicrobial activity of Citrox ${ }^{\circledR}$ bioflavonoid preparations against oral microorganisms. Br. Dent. J. 2011, 210, E22. [CrossRef] [PubMed] 